\title{
Decentralized Resource Allocation Mechanisms in Networks: Realization and Implementation
}

\author{
Tudor Mihai Stoenescu ${ }^{1}$ and Demosthenis Teneketzis ${ }^{2}$ \\ 1 Social and Information Science Laboratory \\ California Institute of Technology \\ tudorecaltech.edu \\ 2 Department of Electrical Engineering and Computer Science \\ University of Michigan \\ teneketzis@eecs.umich.edu
}

\begin{abstract}
Summary. We discuss how decentralized network resource allocation problems fit within the context of mechanism design (realization theory and implementation theory), and how mechanism design can provide useful insight into the nature of decentralized network resource allocation problems. The discussion is guided by the unicast problem with routing and Quality of Service (QoS) requirements, and the multi-rate multicast service provisioning problem in networks. For these problems we present decentralized resource allocation mechanisms that achieve the solution of the corresponding centralized resource allocation problem and are informationally efficient. We show how the aforementioned mechanisms can be embedded into the general framework of realization theory, and indicate how realization theory can be used to establish the mechanisms' informational efficiency in certain instances. We also present a conjecture related to implementation in Nash equilibria of the optimal centralized solution of the unicast service provisioning problem.
\end{abstract}

\subsection{Introduction: Motivation and Challenges}

Today's fast paced world requires a vast amount of information exchange in order to operate efficiently. With the various technological advances the number of types of services being offered (e.g. telephone connections, live audio broadcasting, live video broadcasting, library database access, e-mail, world wide web), is constantly increasing. Each type of service imposes different Quality of Service (QoS) requirements (e.g. delay, percentage of data packet loss, jitter) on the delivery methods. To address these needs extensive communication networks were developed in the past century. Many of these networks (such as telephone networks) were initially designed for the delivery of certain types of information and were later adapted to accommodate new information exchange needs.

Most of today's networks, called integrated services networks, support the delivery of a variety of services to their users. One of the main challenges in integrated services networks is the design of resource allocation strategies which guarantee the 
delivery of different services, each with its own QoS requirement, and maximize some performance criterion (e.g. the network's utility to its users). The challenge in determining such resource allocation strategies comes from the fact that the network is an informationally decentralized system.

The topic of resource allocation for informationally decentralized systems has been explored in great detail by mathematical economists in the context of mechanism design. In this chapter we discuss how decentralized network resource allocation problems fit within the context of mechanism design, and how ideas from mechanism design can provide useful insight into the nature of decentralized network resource allocation problems. We first present a brief history of the development of the ideas that led to the current state-of-the-art of the theory of mechanism design (Section 13.2.1). Then, we present the key features of the two components of mechanism design, namely, realization theory (Section 13.2.2) and implementation theory (Section 13.2.3). To illustrate how network resource allocation problems fit within the context of mechanism design, and how mechanism design can be used to provide insight into the nature of network resource allocation problems, we consider two classes of network problems: unicast service provisioning with routing and QoS requirements, and multi-rate multicast service provisioning. We discuss these problems in Section 13.3 from the realization theory point of view. We investigate unicast resource allocation with routing from the implementation theory point of view in Section 13.4. We conclude in Section 13.5, by summarizing our discussion and identifying some open problems.

\subsection{Mechanism Design}

\subsubsection{Historical background}

Traditionally, economic analysis treated economic systems as one of the "givens." That is, it was assumed that for a given problem the structure of the economic system considered in order to generate a solution is fixed.

At the turn of the last century, economists started to question the effect that the structure of the system has upon the solution of the problem. Although the search for a "better system" has been around at least since Plato's Republic, this issue became more relevant with the emergence of the socialist and capitalist economic systems.

One of the major issues that arose from the debate surrounding the virtues of the socialist and the capitalist systems was the methodology in which resources should be allocated. From the early stages of the debate most economists envisioned that the resources in a socialist system would be allocated by the use of a centralized coordinator, while in a capitalist system the resources would generally be allocated through the use of a market. This debate attracted a lot of attention, with prominent economists like Bukharin [15, 16], Dickinson [26], Doob [29], Kautsky [61], Lange [70], Lenin [72], Lerner [73, 74], Marschak [80], Neurath [93], and Taylor [130] arguing in favor of the socialist system; Pierson [105], von Hayek [135-139], and L. von Mises [140] arguing in favor of the capitalist system; and Barone [10], 
Pareto[99-101], and Walras [141] contributing to the mathematical foundations. ${ }^{3}$ Yet, with all these contributions, very few fundamental results on resource allocation theory were available until the 1930s. One of the major reasons for this was the lack of mathematical tools required to tackle such problems. The research efforts in the 1930s along with the subsequent mathematization of classical welfare economics, von Hayek's work, and the developments on mathematical programming and game theory set the mathematical foundations for the development of the theory of mechanism design.

In the 1930s three major research efforts relevant to the design of allocation mechanisms began: i) the development of resource allocation methods for the socialist economy (with major contributors being Lange [70], Lerner [73, 74] and Taylor [130]); ii) the efforts of Hotelling [37, 38] and Lerner [73] on marginal cost pricing and consumer-producer surplus; and iii) the development of the "new welfare economics" (with major contributions by Hicks [36], Kaldor [55], and Scitovsky [21]). A decade later researchers began developing the mathematization of "classical welfare economics" [3, 4, 7, 9, 24, 25, 66, 71].

In the late 1930s and early 1940s, von Hayek made the following key observations: (i) the amount of available information required and the amount of calculations needed by a central-control system in order to determine an optimal allocation would be enormous; and (ii) the economic incentives provided by the market economy could not be reproduced by any of the socialist models. Von Hayek argued that even with the use of "fast" algorithms, the problem required to be solved may be overwhelming and no human or computer could calculate a solution. Von Hayek also argued that the process of placing the "right" information in the hands of the computing and decision making agencies may be very difficult. Since information is dispersed throughout the economy (with no agent having full knowledge of the state of the economy), it must be communicated among the economic agents in order for a solution to be determined. This information exchange may be very costly, and in many cases it may be impossible for a central-control agent to have full knowledge of the state of the economy.

Following the initial results of the 1930s, three major lines of research had a great influence on the development of resource allocation mechanisms and helped to establish a more comprehensive understanding of the main features of such problems:

- activity analysis and linear programming (Dantzig, Kantorovich, Koopmans),

- game theory and iterative solution procedures (von Neumann and Morgenstern, George Brown, Julia Robinson),

- investigation of the relationship between linear/nonlinear programming, two person zero-sum games and Lagrange multipliers (Gale, Kuhn, Tucker).

The early breakthroughs in the field of linear programming greatly influenced the mathematization of classical welfare economics. Although linear programming models are not able to handle goal conflicts due to the multiplicity of consumers as

\footnotetext{
${ }^{3}$ For a more detailed presentation of the historical aspects of the socialist controversy we refer the reader to [125].
} 
well as constraints arising from the decentralization of information, they are still a very important step in analyzing and understanding multi-objective problems.

Game theory is concerned with the interactive behavior of "rational" man. It is the study of mathematical models of conflict among rational decision makers. Game theory provided general mathematical techniques for analyzing situations where two or more decision makers' decisions influence one another's welfare. As such, game theory offered insights of fundamental importance for researchers in many branches of social sciences and technology, including resource allocation mechanisms.

Understanding the interplay between mathematical programming techniques and zero sum games, along with the use of Lagrange multipliers (interpreted as shadow prices in economic systems) helped to develop tools for analyzing general mechanism design problems and provided a better perspective on solution methods.

The original work in classical welfare economics, von Hayek's observations, and the development of linear programming, game theory and Lagrange multipliers, set the foundations for the formal development of mechanism design in terms of both the "realization" and the "implementation" of Social Choice Rules (SRC's) (goal correspondences) by decentralized economic systems. Realization theory and implementation theory are the two basic components of mechanism design. We briefly present the key features of realization and implementation theory next.

\subsubsection{Realization theory}

Formally, resource allocation problems can be described by the following triple: environment, action space, and goal correspondence. We define the environment $\mathbf{E}$ of such problems to be the set of individual endowments, the technology, and preferences, taken together. More generally, the environment is defined as the set of circumstances that cannot be changed either by the designer of the mechanism or by the agents. The action space $\mathbf{A}$ of the problem is considered to be the set of all possible actions, (e.g. resource exchanges) conducted by the various agents. Finally, the goal correspondence $\pi$ is the map from $\mathbf{E}$ to $\mathbf{A}$ which assigns for every $e \in \mathbf{E}$ the set of actions in $\mathbf{A}$ which are solutions to the resource allocation problem.

The setup described above corresponds to the case in which one of the agents has enough information about the environment so as to determine the actions that would satisfy the goal correspondence (i.e. the information in the systems is centralized). Generally this is not the case. Usually, different agents have different information about the environment (i.e. we have an informationally decentralized system). For this reason it is desired/necessary to devise a message exchange process among the various agents that eventually enables them to jointly take an action which corresponds to a solution of the centralized problem. We call such a process of communication, decisions and actions a resource allocation mechanism.

The function of a resource allocation mechanism is to guide the agents (economic or otherwise) to make decisions that determine the flow of resources. More specifically, mechanisms provide rules, called response rules (or communication rules), according to which agents communicate messages to other agents. These messages 
are generally generated based on the agents" "private" information about the environment and prior messages received from other agents. To provide for a transition from the dialogue to decisions and actions, the mechanism must also have an outcome rule which specifies what actions are to be taken given the course of the dialogue. Generally, the mechanism rules may be deterministic or probabilistic; mathematically they are expressed as functions or correspondences.

For simplicity we are going to consider the case in which our mechanism can be represented by a tâtonnement process. This process consists of a communication stage in which agents exchange formal messages in an iterative fashion, followed by a decision process, and finally a translation of decisions into actions. The case in which the communication, decisions, and actions overlap in time (a non-tâtonnement process) require more general theory and will not be discussed here.

The first effort to formally study resource allocation mechanisms can be traced back to Hurwicz's work [40-43]. Hurwicz models the communication process by means of a language and response functions, specifying how each agent determines the message to be emitted at each stage of the iterative exchange of messages. After the process of communication terminates, decisions are determined on the basis of the state of information at the final stage of communication.

Formally, the mechanism model proposed by Hurwicz can be described by the triple $(\mathcal{M}, \mu, h)$ : a message space $\mathcal{M}$, an equilibrium message correspondence $\mu$, and an outcome correspondence $h$. The message space is the set of messages that may be exchanged by the agents. The equilibrium message correspondence describes the sets of messages that the agents "agree" upon given any particular environment. The outcome function describes the set of actions that are taken based on a particular set of "equilibrium" messages. The formulation above is depicted graphically in Fig. 13.1 (cf. [110]).

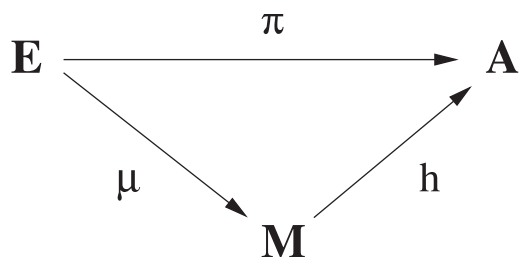

Fig. 13.1. Message exchange for a decentralized system.

Realization theory is concerned with the existence and design of mechanisms $(\mathcal{M}, \mu, h)$ such that the diagram in Fig. 13.1 commutes. Hurwicz's setup is quite general and can incorporate many types of mechanisms. Given a specified goal correspondence (alternatively called social choice rule or social welfare correspondence) there may be several mechanisms $(\mathcal{M}, \mu, h)$ such that the diagram of Fig. $13.1 \mathrm{com}$ mutes. Each of these mechanisms may have different "communication" and "information processing" characteristics. For example, in a market mechanism with an "auctioneer" the messages exchanged could be prices and demands. In this model 
the auctioneer updates the prices according to the "excess demand" while the agents update their demands based on the prices. In such a situation the message space $\mathcal{M}$ is small but the "information processing" required until a final action is taken is large, mainly because the process is iterative and the number of required iterations may be very large (in theory they may be infinite). On the other hand, in a "central command" type of mechanism the messages are the signals the agents send to a "central authority" so as to describe their environments. After receiving the agents' messages, the central authority calculates an optimal allocation of resources and sends the order for action to the agents. In this situation, if the space of environments is "rich" the "dimensionality" [69] of the message space required for communication is very large. On the contrary, the amount of information processing is small because it takes only one iteration to implement a centralized decision.

The characterization and classification of mechanisms in terms of their "communication" and "information processing" requirements is an open research area. So far, research concentrated mainly on the "communication" requirements specifically on the "dimensionality" [39-44, 81, 88] of the message space $\mathcal{M}$ required so that the diagram in Fig. 13.1 should commute. Mechanisms $(\mathcal{M}, \mu, h)$ that posses the aforementioned commutative property, have a message space $\mathcal{M}$ of minimum "dimensionality," and satisfy some additional requirements (described below), have been called "informationally efficient." The characterization and comparison of mechanisms according to their "information processing" requirements has received very little attention. In the sequel we will state and discuss more precisely the conditions under which "realization" theory was developed.

The following requirements are generally imposed on Hurwicz's models:

R1. For each element of the environment $e \in \mathbf{E}$ there exists a non-empty set of possible feasible actions. The notion of feasibility can usually be split into two categories individual feasibility and compatibility. In particular:

1. In standard models of production economies, an agent's individually feasible actions are defined to be the set of actions formed by the agent's production function. Within the context of the network problems considered in Section 13.3 a user's individual feasible actions are formed by the set of non-negative demand vectors. On the other hand, the network's individual feasible actions are formed by the set of amounts of services that are delivered and satisfy certain QoS requirements and the network's capacity constraints.

2. We call an action incompatible if given two different input-output vectors of two agents, one calls for an input which the other does not propose to supply. Within the context of the network problems considered in Section 13.3 an action is incompatible if in equilibrium a user requests an amount of service which differs from the amount of service the network intends to supply.

R2. For each element of the environment the set of feasible actions that satisfy the goal correspondence is non-empty.

R3. The actions generated by $\pi$ must satisfy some sort of optimality criterion. Examples of such criteria are: efficiency of production (defined by Koopmans [65]), 
optimality (introduced by Pareto [100] under the name of "ophelimity"4 maximizing), and socially welfare maximizing (defined by Bergson [13], Samuelson [113] and Arrow [6, 8]).

R4. For any environment $e \in \mathbf{E}, \mu(e) \neq \emptyset$; that is, for any environment there exists a set of messages to which all agents "agree."

R5. The maps $\pi, \mu$ and $h$ satisfy the following relationship:

$$
h(\mu(e)) \subseteq \pi(e) \quad \forall e \in \mathbf{E} .
$$

In other words, for any system environment, in equilibrium, the messages exchanged by the system agents enable the agents to take actions which achieve an optimal centralized solution. Mechanisms satisfying this assumption are also referred to as non-wasteful.

R6. The non-wasteful criterion established above can sometimes be inadequate. For example we can have the case where the equilibria of a given process always favors one group of participants at the expense of others. We call such mechanisms biased. To avoid biased mechanisms we require unbiasedness.

A formal test for unbiasedness can be viewed as follows: Suppose that we think of our process as being formed of two stages. In the first stage the process "distributes parameters" (e.g. resources, information etc.) to the various agents, while in the second stage we have a tâtonnement process. If for any environment $e \in \mathbf{E}$ and any goal realizing action $a \in \pi(e)$ there exists a set of distributional parameters such that at the end of the tâtonnement process the agents take action $a$, then the process is called unbiased.

R7. For any environment, the rules of the process lead the system to a uniquely determined allocation. This requirement may be difficult to satisfy even in the case of market-based economies. In such economies there may be multiple allocations which are optimal for a fixed set of prices, however, all of these allocations have the same utility for all the agents. We call such processes, where equilibrium indeterminacies are trivial in nature, essential single-valued.

R8. There are two types of information regarding the environment agents have access to: direct and indirect. The agent's direct information is obtained through observations of the environment. The indirect information is gathered by the agent through the exchange of messages with other agents. We assume that an agents' direct information is information pertaining only to himself and not to other agents. We will refer to processes satisfying this property as informationally consistent.

When considering the equilibrium messages generated by agents, we call a process privacy preserving when all the agents generate their messages based only on

\footnotetext{
${ }^{4}$ Pareto was troubled with the concept of 'utility.' In its common usage utility meant the well-being of the individual or society. Pareto realized that when people make economic decisions they are guided by what they think is desirable for them whether or not that corresponds to their well-being. Thus, he introduced the term "ophelimity" to replace the worn-out 'utility.' Later, preferences replaced Pareto's ophelimity.
} 
their own information about the environment. Hurwicz's model restricts attention to privacy preserving resource allocation mechanisms.

R9. Assume that the agents communicate with one another through a communication alphabet which permits them to communicate in one shot their direct information (profile) to the other agents. Such a language in most cases is too complex for consideration and hence is undesirable. Restricting the language may also not be enough to alleviate this problem. Agents may be able to encode in a relatively "simple" language their full profile. This type of encoding though may be done by the use of equilibrium message and outcome correspondences which are highly discontinuous and resemble such functions as the Peano space filling curves [5]. Such mechanisms are generally highly unstable (hence undesirable) since minor perturbations/errors in communication will lead to drastically different/nonoptimal actions. In order to alleviate problems such as the above, we introduce the following requirement: We impose extra conditions, such as spot threadiness, on the correspondences $\mu$ and $h$.

Definition 1. A correspondence $F: E \rightarrow M$ is spot threaded if for every $e \in E$ there exists an open set $U_{e} \subseteq E$, and a continuous function $f: U_{e} \rightarrow M$ such that $f\left(e^{\prime}\right) \in F\left(e^{\prime}\right)$ for all $e^{\prime} \in U_{e}$.

We note that the first three requirements $\mathbf{R} \mathbf{1}-\mathbf{R} 3$ are constraints on the type of problems considered, and they are defined independently of the mechanism. The

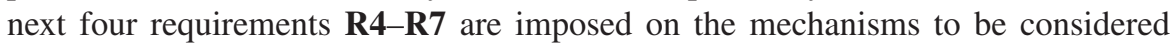
and are generally referred to as (Pareto) satisfactoriness ${ }^{5}$. Mechanisms that satisfy R8-R9 are called regular.

Realization theory was developed using subsets of the requirements above. To proceed with a more formal description of the results on communication and information processing requirements we need the following definitions:

Definition 2. We say a mechanism $(\mathcal{M}, \mu, h)$ is goal realizing if it satisfies requirements R1, R2, R4 and $\mathbf{R 5}$.

Definition 3. We say that a mechanism $(\mathcal{M}, \mu, h)$ is informationally efficient if it is goal realizing and regular and it has a message space of a dimensionality which is minimal among all the other goal realizing and regular mechanisms.

To the best of our knowledge, this definition of informational efficiency is different from that appearing in most of the literature on realization theory. Our definition, compared to the definition appearing in the literature, imposes more requirements on the properties an informationally efficient mechanism must satisfy. We show that the decentralized network resource allocation mechanisms we present in Section 13.3 are informationally efficient according to our definition of informational efficiency.

Most of the research on realization theory has dealt with the discovery of goal realizing mechanisms that have a message space of minimum dimension among the message spaces of goal realizing and regular mechanisms. Some of the key results

\footnotetext{
${ }^{5}$ In many models requirement $\mathbf{R} 7$ is omitted in the definition of Pareto satisfactoriness.
} 
are: (i) the competitive process is Pareto satisfactory over classical environments ${ }^{6}$ $[88,89]$; (ii) for classical environments, the competitive mechanisms are goal realizing and have a message space of minimum dimension among the message spaces of goal realizing and regular mechanisms $[40,44,88]^{7}$; (iii) competitive mechanisms are informationally efficient for classical environments where the utility functions are of the Douglas Cobb form [88]; (iv) for environments with public goods the Lindahl mechanism is goal-realizing and has a message space of minimum dimension among the message spaces of all goal-realizing and regular mechanisms [43, and references therein]; (v) if the dynamics of allocation mechanisms are considered explicitly and stability is required then the size of the message space has to increase $[53,90]$.

Work in [56, 108, 109] addressed issues related to the complexity of information processing of goal-realizing mechanisms that have message spaces of minimum dimension among the message spaces of goal-realizing and regular mechanisms.

\subsubsection{Implementation theory}

It is well known that the theory of organizational control systems is concerned with two types of rules: operational and enforcement. The operational rules describe how the system "should" operate, while the enforcement rules assure that the operational rules are followed. Enforcement rules fall within two categories: explicit and implicit. While explicit enforcement rules generally use monitoring techniques in order to control agents' behavior in a system, implicit enforcement motivates agents behavior by providing appropriate incentives.

In the previous section we defined a mechanism to be a set of operational rules, according to which the system's agents generate messages which lead to desired actions. The question that arises is: Can we expect the agents to follow such rules? The answer to the question above is provided by the theory of implementation.

The theory of implementation is generally concerned with strategic behavior of allocation procedures, and generally studies implicit enforcing rules. It is concerned with the design/discovery of "game forms" that implement, in some behavioral equilibrium (solution concept), social choice rules/goal correspondences.

Specifically, $N$-agent "game forms" are defined as pairs of the form $(\mathcal{M}, h)$, where $\mathcal{M}=\prod_{i=1}^{N} \mathcal{M}_{i}, \mathcal{M}_{i}$ is the message space of agent $i, i=1,2, \ldots, N$, and $h: \mathcal{M} \rightarrow \mathbf{A}$. Thus, for each profile $m:=\left(m_{1}, m_{2}, \ldots, m_{N}\right)$ of messages, $h(m) \in \mathbf{A}$ represents the resulting outcome or allocation. A game form is different from a game as the consequence of a profile of messages is an outcome (allocation) rather than a vector of utility payoffs. Once a preference profile, i.e. a complete, binary and reflexive preordering $\mathcal{R}\left(e_{i}\right)$, that describes the $\mathrm{i}^{\text {th }}$ agent's preferences over alternatives in $\mathbf{A}$ when i's environment is $e_{i} \in \mathbf{E}_{i}$, is specified for each $i=1,2, \ldots, N$, a game form induces a game.

\footnotetext{
${ }^{6}$ A classical environment is defined to be a convex economy (i.e. concave utility functions and convex constraint sets), free of externalities (an externality is present when wellbeing of an agent is directly affected by the actions of another agent).

${ }^{7}$ In $[40,44,88]$ it is not required that the competitive mechanism should be regular in the whole space of environments.
} 
The principal difference between a game form and Hurwicz's original model, described by $(\mathcal{M}, \mu, h)$, is the following: In game forms the message correspondence (described by $\mu$ in Hurwicz's model) is not a design variable, but is induced by the outcome function and the behavioral equilibrium concept (e.g. Nash, Bayesian Nash, maxmin, undominated etc.).

A solution concept (or equilibrium concept) specifies the strategic behaviors of agents (individuals, users) faced with a game form $(\mathcal{M}, h)$ given a preference profile $\mathcal{R}(e):=\left(\mathcal{R}\left(e_{1}\right), \mathcal{R}\left(e_{2}\right), \ldots, \mathcal{R}\left(e_{N}\right)\right)$. Hence, a solution concept is a correspondence $\Lambda$ that identifies a subset of $\mathcal{M}$ for any given specification $(\mathcal{M}, h, \mathcal{R}(e))$. We define

$$
\mathfrak{Q}_{\Lambda}:=\{a \in \mathbf{A} \mid \exists m \in \Lambda((\mathcal{M}, h, \mathcal{R}(e))) \text { s.t. } h(m)=a\}
$$

as the set of outcomes associated with the solution concept $\Lambda$.

To illustrate (13.2) consider a pure strategy Nash equilibrium as the solution concept. For any given $(\mathcal{M}, h, \mathcal{R}(e))$ a pure Nash equilibrium is a message $m:=$ $\left(m_{1}, m_{2}, \ldots, m_{N}\right) \in \mathcal{M}$ such that

$$
h(m) \mathcal{R}\left(e_{i}\right) h\left(\bar{m}_{i}, m_{-i}\right)
$$

for all $i=1,2, \ldots, N$, and all $\bar{m}_{i} \in \mathcal{M}_{i}$, where

$$
m_{-i}:=\left(m_{1}, m_{2}, \ldots, m_{i-1}, m_{i+1}, \ldots, m_{n}\right) .
$$

Denote the messages satisfying (13.2) by $\mathbf{N E}((\mathcal{M}, h, \mathcal{R}(e)))$. Then the set of associated outcomes is

$$
\mathfrak{Q}_{\mathbf{N E}}((\mathcal{M}, h, \mathcal{R}(e))):=\{a \in \mathbf{A} \mid \exists m \in \mathbf{N E}((\mathcal{M}, h, \mathcal{R}(e))) \text { s.t. } h(m)=a\} .
$$

To precisely define how social choice correspondences are implicitly enforced via game forms in some behavioral equilibrium we need the following:

Definition 4. A social choice correspondence $\pi: \mathbf{E} \rightarrow \mathbf{A}$ is implemented by the game form $(\mathcal{M}, h)$ via the solution concept $\Lambda$ if

$$
\mathfrak{Q}_{\Lambda}((\mathcal{M}, h, \mathcal{R}(e)))=\pi(e)
$$

for all $e \in \mathbf{E}$.

Definition 5. A social choice correspondence $\pi: \mathbf{E} \rightarrow \mathbf{A}$ is said to be implementable via the solution concept $\mathfrak{Q}$ if there exists a game form $(\mathcal{M}, h)$ that implements it.

The form of implementation above is called full implementation or strong implementation since it requires that the outcomes of a game form coincide with those of the social choice correspondence. A weaker form of implementation (called weak implementation) is one where for every $e \in \mathbf{E}$,

$$
\mathfrak{Q}_{\Lambda}((\mathcal{M}, h, \mathcal{R}(e))) \subseteq \pi(e) .
$$


A comparison between full implementation and weak implementation is presented in Thompson [133].

Within the context of implementation theory there have been significant developments in the characterization of social choice rules that can be implemented in dominant strategies [20, 32]; in Nash equilibria [83-85, 112, 143]; or in refined Nash equilibria such as subgame perfect equilibria [2, 87], undominated Nash equilibria $[1,46,48,96]$, trembling hand perfect Nash equilibria [123]; or in Bayesian Nash equilibria [45, 95, 97, 106]. Excellent survey articles on implementation theory are $[47,85,94]$. These articles summarize the state of the art on implementation theory up to the time of their publication.

Direct revelation game forms (otherwise called direct mechanisms or direct revelation mechanisms) is a particular class of game forms that have a natural appeal and have received significant attention. In direct revelation game forms $M_{i}=E_{i}$ for each agent $i$. In effect then each agent reports his own environment, but not necessarily his true one. The interest in direct revelation game forms stems from the revelation principle. The revelation principle is the observation that if a game form $(M, h)$ implements a social choice correspondence $\pi(\pi: E \rightarrow A)$, then there exists a direct revelation game form $\left(E, h^{*}\right)$ which has the following properties: (1) announcing one's true characteristic is an equilibrium message; and (2) $h^{*}\left(e_{1}, e_{2}, \ldots, e_{N}\right)=h^{*}(e) \in \pi(e)$ for all $e \in E$. Even though the direct revelation game form has the aforementioned properties, it does not necessarily implement the social choice correspondence $\pi$. This is because the direct mechanism may have multiple equilibria which give rise to outcomes which, for some $e \in E$ are not in $\pi(e)$ (see [20]). Thus, one cannot conclude from the revelation principle that all one ever needs to consider are direct revelation game forms. Only under certain conditions (see [20]) a social choice rule $\pi$ can be implemented by a direct revelation game form. Most of the literature on implementation in dominant strategies and in Bayesian equilibria has used truthful implementation, an implementation concept that requires only that the truthful equilibrium of a direct revelation game form $\left(E, h^{*}\right)$ be in the choice set, i.e., $h^{*}(e) \in \pi(e) \forall e \in E$.

\subsection{Mechanism Design in Networks: A Realization Theory Point of View}

To illustrate how mechanism design can be used in networks we present two classes of network resource allocation problems and discuss them from the realization theory point of view. The two classes of problems are: 1) resource allocation in unicast with routing and end-to-end Quality of Service requirements; and 2) rate allocation in multi-rate multicast service provisioning. For these two problems we present two distinct pricing mechanisms which achieve the solution of the centralized resource allocation problem, satisfy the informational constraints imposed by the decentralization of information in networks, and are informationally efficient. We show how these mechanisms can be embedded into Hurwicz's abstract frame- 
work; in [125] we have shown how ideas from realization theory can be used to establish the pricing mechanisms' informational efficiency.

\subsubsection{Unicast with routing and Quality of Service requirement}

\section{Problem formulation}

We consider a set of users/agents, denoted by $\mathbf{N}=\{1,2, \ldots, N\}$, requesting various services from a network. For each user $i \in \mathbf{N}$ we denote by $\mathbf{M}_{i}$ the set of types of services requested by that user. For each $i \in \mathbf{N}$ each service $j \in \mathbf{M}_{i}$ must satisfy some sort of end-to-end Quality of Service (QoS) requirements denoted by $\mathcal{F}_{i j}$. Assume that user i's preference over the set of services it requests is summarized by a utility function $U_{i}\left(\bar{x}_{i}\right)$, where $\bar{x}_{i} \in \mathbb{R}_{+}^{\mathbf{M}_{i}}$. We consider the network to be the $(N+1)^{t h}$ agent.

The network is formed by a set of links $\mathbf{L}$. For every $l \in \mathbf{L}, \mathbf{K}_{l}$ denotes the set of resources on link $l$. Denote by $\mathbf{K} \triangleq \bigotimes_{l \in \mathbf{L}} \mathbf{K}_{l}$ the set of resources available at the different links of the network, and by the vector $c_{\mathbf{K}}$ the amount resources at those links. Define $\mathbf{T}_{\mathbf{L}}$ to be the topology of the network and $\mathbf{R}_{\mathbf{T}_{\mathbf{L}}}$ to be the set of possible routes over which each service requested from the network can be delivered. For each user $i \in \mathbf{N}$ and for each service type $j \in \mathbf{M}_{i}$ denote by $\mathbb{F}_{i j}\left(\mathbf{R}_{\mathbf{T}_{\mathbf{L}}}, \mathbf{K}\right)$ the set of all resource allocations along all the possible routes of service $j$ that guarantee the end-to-end QoS requirements $\mathcal{F}_{i j}$. Also, for $t \in \mathbf{R}_{\mathbf{T}_{\mathbf{L}}}, i \in \mathbf{N}$, and $j \in \mathbf{M}_{i}$ denote by $\mathbb{F}_{i j t}\left(\mathbf{R}_{\mathbf{T}_{\mathbf{L}}}, \mathbf{K}\right)$ the set of all resource allocations along route $t$ that guarantee the end-to-end QoS requirements $\mathcal{F}_{i j}$.

The goal of the network is to allocate resources to the various services in order to maximize a social welfare function described by the sum of the user utilities, while satisfying the QoS requirements imposed by the offered services. Hence, the goal of the network is:

$$
\max _{x, \mathbf{R}_{\mathbf{T}_{\mathbf{L}}}} \sum_{i \in \mathbf{N}} U_{i}\left(\bar{x}_{i}\right)
$$

subject to:

$$
\begin{array}{cc}
\bar{x}_{i} \in \mathbb{R}_{+}^{\mathbf{M}_{i}} ; & \text { P.a } \\
r^{i, j} \in \mathbb{F}_{i j}\left(\mathbf{R}_{\mathbf{T}_{\mathbf{L}}}, \mathbf{K}\right) ; & \text { P.b } \\
\sum_{i \in \mathbf{N}} \sum_{j \in \mathbf{M}_{i}} x_{j}^{i} r_{l, k}^{i, j} \leq c_{l, k} ; & \text { P.c }
\end{array}
$$

For each user $i \in \mathbf{N}$, each service $j \in \mathbf{M}_{i}$, any set of routes $\mathbf{R}_{\mathbf{T}_{\mathbf{L}}}$ and any resource availability $\mathbf{K}$, the set $\mathbb{F}_{i j}\left(\mathbf{R}_{\mathbf{T}_{\mathbf{L}}}, \mathbf{K}\right)$ is well defined, compact and non-empty; P.d

The users' utility functions are concave, strictly increasing and continuously differentiable.

In P.b and P.c. $r_{l, k}^{i, j}$ stands for the amount of resource of type $k$ on link $l$ assigned to the service of type $j$ requested by user $i, r^{i, j}$ represents the vector of resources allocated to user $i$ for service $j$, and $c_{l, k}$ is the amount of resource of type $k \in \mathbf{K}_{l}$. 
In addition, the following informational constraints are present:

The network has no information about the users' utility functions or the number of users interested in services.

Each user's preferences over the particular services is his private information. The users are unaware of the topology of the network, the amount of resources available on each link, and method by which the network delivers their services. The users are also unaware of the number of other users requesting services from the network, or their utility functions.

The assumption that the network manager has complete knowledge of the network topology and resources is not an unrealistic one. For example, a corporate intranet or VPN (virtual private network) may have a single provider of resources and services, who is likely to have such knowledge about the network, and who will assume the roll of network management in collecting aggregate excess demand on links and adjusting link prices. In particular, some resource/service providers use very sophisticated network management tools to monitor in real time the proper functions of a network (e.g., events such as congestion, fault, server ups and downs), and to issue appropriate response/commands. Such monitoring requires complete knowledge of the network (e.g., topology, resources, router/link capacities), as well as separate network management protocols to pass information to and from the management site. These tools can easily be used to acquire information on aggregate excess demands and to adjust link prices.

The goal in unicast with routing and QoS requirements is to determine a mechanism that allocates resources in order to generate services for individual users, satisfies the QoS requirements for all the services delivered, is social welfare maximizing $^{8}$, and satisfies the aforementioned informational constraints. To achieve this goal we present a market mechanism, which results in a solution of the centralized optimization problem $\mathbf{P}-\mathbf{P . e}$ and satisfies the informational constraints P.f-P.g.

Unicast service provisioning has received significant attention. Most of the results on decentralized resource allocation in unicast service provisioning, currently available in the literature are based on pricing mechanisms $[17,19,22,33,49,54$, $62,64,76,78,79,91,92,103,104,131,142]$. These publications have addressed, either by analysis $[19,22,33,49,62,64,76,78,92,131,142]$, or simulation and analysis [17, 33, 91, 103, 104], a subset of the issues outlined in the goal of the unicast problem stated in the previous paragraph. A significant number of publications have dealt with single link networks $[22,91,104,142]$, or with the allocation of a single resource per connection [22, 33, 49, 64, 78, 91, 92, 104, 142].

\section{Market mechanism}

We proceed as follows: First, we describe a competitive market economy consisting of service providers, users and an auctioneer. Then, within the context of this

\footnotetext{
${ }^{8}$ The social welfare function in this work is characterized by the sum of individual user utility functions.
} 
market we specify a procedure, used by the auctioneer, which leads to a resource allocation that achieves a solution of Problem $\mathbf{P}$.

\section{Description of the market}

In our market, for conceptual clarity, we assume that the network consists of a service provider and an auctioneer. Under this assumption, the economy consists of the following three types of agents: a service provider, users and an auctioneer. The auctioneer sets the prices per unit of resource at each link. The price of resource $k$ at link $l$ is denoted by $\lambda_{l, k}$. The service provider and the users are price takers. They act as if their behavior has no effect on the equilibrium prices reached by the market allocation process. The service provider uses the network's resources and the prices $\lambda_{l, k}$, specified by the auctioneer, to set up services and the corresponding prices for each unit of these services. Then, it announces the price per unit of service for each service to the users. Based on the announced prices, each user decides the type of services and the amount of each service it should request.

We observe that the price taking assumption and the fact that we try to maximize the sum of the users' utilities imply that: (i) the service providers will not attempt to make a profit; and (ii) the service prices are directly derived from the resource prices.

Below we describe each type of agent in more detail.

Service providers: The users request services from the service providers. Each of these requests is described by the origin, destination and the minimal level of quality of service required. The services are indexed by the $(i, j)$ pair, with $i \in \mathbf{N}$ representing the user and $j \in \mathbf{M}_{i}$ representing the service type. For each pair $(i, j)$ there exists a set $\mathbf{T}^{i, j}$ of possible routes that can be used. Denote by $\mathbf{V}_{i j t}$ the set of links forming route $t \in \mathbf{T}^{i, j}$. The service provider allocates resources $r^{i, j, t}(\lambda) \in \mathbf{V}_{i j t}$ so that the minimum cost for the service and the lowest acceptable level of quality of service are attained. We assume that each service can not be distributed over multiple routes.

Since the service provider is not a profit maker, it allocates resources for each type of connection by solving:

$$
r^{i, j, t}(\lambda) \in \underset{r^{i, j, t} \in \mathbf{F}^{i, j, t}}{\operatorname{argmin}} \sum_{l \in \mathbf{V}_{i j t}} \sum_{k \in \mathbf{K}} \lambda_{l, k} r_{l, k}^{i, j, t}
$$

where $i \in \mathbf{N}, j \in \mathbf{M}_{i}, t \in \mathbf{T}^{i, j}$. For each $(i, j)$ pair, equation (13.5) generates a set of allocations that result in a minimum price per unit of service for each route $t \in \mathbf{T}^{i, j}$. Then, the service provider computes the price per unit of service for route $t$,

$$
p_{j, t}^{i}(\lambda)=\sum_{l \in \mathbf{V}_{i j t}} \sum_{k \in \mathbf{K}} \lambda_{l, k} r_{l, k}^{i, j, t}
$$

where $r_{l, k}^{i, j, t}$ are determined by (13.5). Finally, the service provider computes, for each $i \in \mathbf{N}, j \in \mathbf{M}_{i}, \quad p_{j}^{i}(\lambda)=\min _{t \in \mathbf{T}^{i, j}} p_{j . t}^{i}(\lambda)$ 
and announces the prices $p_{j}^{i}(\lambda), i \in \mathbf{N}, j \in \mathbf{M}_{i}$, to the users. If for some $(i, j)$ there are two or more routes of minimum price, the service provider picks one of these routes.

Users: Users request one way connections from the service provider. Based on the price $p_{j}^{i}(\lambda)$, announced by the service provider, the users demand a number of connections determined by

$$
x^{i}(p) \in \underset{x^{i} \in \mathbf{X}^{i}}{\operatorname{argmax}}\left[u_{i}\left(x^{i}\right)-\sum_{j \in \mathbf{M}_{i}} x_{j}^{i} p_{j}^{i}(\lambda)\right], \quad \forall i \in \mathbf{N} .
$$

Auctioneer: The role of the auctioneer is to regulate the prices of the resources. He does this based on the aggregate excess demand vector $z(\lambda, \mathbf{t})$ :

$$
z_{l, k}(\lambda, \mathbf{t}) \triangleq \sum_{i \in \mathbf{N}} \sum_{j \in \mathbf{M}_{i}}\left(x_{j}^{i}(\lambda) r_{l, k}^{i, j, t}(\lambda)\right)-c_{l, k}
$$

where $l \in \mathbf{L}, k \in \mathbf{K}$, and $r_{l, k}^{i, j, t}$ is determined by (13.5)-(13.7).

\section{The tâtonnement process}

We present a tâtonnement process, specified by an algorithm, called Algorithm 1, that describes how the market works. The algorithm proceeds iteratively as follows:

Step 1: The auctioneer announces prices $\lambda$ for the resources at each node of the network. The users announce their desired services to the service provider.

Step 2: Based on the auctioneer's announcement, the service provider computes the minimum price per unit of service according to (13.5)-(13.7). The service provider announces these prices to the users.

Step 3: Based on the prices $p$ announced by the service provider, the users request services in the amount $x(p)$ satisfying (13.8).

Step 4: Based on the service demand vector $x(p)$, the auctioneer computes, through (13.9) the excess demand vector $z(\lambda)$.

Step 5: If $z(\lambda) \leq 0$ then the process ends. Otherwise the auctioneer changes the prices $\lambda$ of resources according to a specific mechanism based on Scarf's algorithm which is described in detail in $[125,129,132]$, announces new prices, say $\lambda^{\prime}$, and the process is repeated from Step 2 on.

\section{Embedding into Hurwicz's framework}

We embed the unicast routing problem, formulated in Section 13.3.1, within the framework of Hurwicz's model described in Section 13.2.2.

\section{The resource allocation problem:}

As presented in Section 13.2.2, a resource allocation problem can be described by the following triple: environment, action space, and goal correspondence. 
Environment: "Characteristics" of a particular agent $i$, say $e^{i}$, is called the local environment of $i$. The set of all possible environments of $i$ is denoted by $E^{i}$. The (system) environment is a tuple consisting of the local environments of all agents and is denoted by $e$. The set of possible system environments is denoted by $E:=\left(\otimes_{i \in \mathbf{N}} E^{i}\right) \otimes E^{N+1}$.

For the network Problem $\mathbf{P}$ the local environment $E^{i}$ of each user $i$ is the set of differentiable concave functions on $\mathbb{R}_{+}^{\mathbf{K}}$. The local environment of the network is the set $E^{N+1} \triangleq\left\{\left\{\mathbf{T}_{\mathbf{L}}\right\} \times\left\{\mathbf{R}_{\mathbf{T}_{\mathbf{L}}}\right\} \times\{\mathbf{K}\} \times \mathbb{F}_{i j}\left(\mathbf{R}_{\mathbf{T}_{\mathbf{L}}}, \mathbf{K}\right) \times\left\{c_{\mathbf{K}}\right\}\right\}$.

Action Space: The set of possible actions taken by the system is called the action space of the system, and is denoted by $\mathbf{A}$. For Problem $\mathbf{P}$ the action space is the feasible region of $\mathbf{P}$.

Goal Correspondence: The relation between the environments and the (desired) actions of the system is represented by a point-to-set map, called the goal correspondence / social choice rule / social welfare maximizing rule, and is denoted by $\pi$. For Problem $\mathbf{P}, \pi: E \rightarrow \mathbf{A}$ is defined as follows:

$$
\pi(e):=\operatorname{argmax} P(e) .
$$

\section{Mechanism specification}

A mechanism in equilibrium correspondence form is characterized by the following triple $(\mathcal{M}, \mu, h)$, where $\mathcal{M}$ is the message space, $\mu$ is the equilibrium correspondence, and $h$ is the outcome function.

Message Space: The set of messages chosen for communication by the designer is called the message space, and is denoted by $\mathcal{M}$. The size of a finite dimensional message space $\mathcal{M}$ is defined to be the dimension [69] of the smallest real vector space in which there is an open set $W$ such that $\mathcal{M} \subseteq W$. The size of $\mathcal{M}$ is denoted by $\operatorname{dim} \mathcal{M}$.

In the allocation mechanism described above (see [129] for details), two types of messages are exchanged among agents:

- The prices per unit of service for each service are communicated by the network to the users. We denote by $\bar{p}:=\left(p_{1}, p_{2}, \ldots, p_{q}\right)$ the vector of prices, where $q$ is the number of network services available for delivery.

- The demands for services communicated by the users to the network. We denote by $\bar{x}:=\left\{x_{j}^{i} \mid i \in \mathbf{N}, j \in \mathbf{M}_{i}\right\}$ the vector of user demands.

Thus, the message space for Problem $\mathbf{P}$ has dimension equal to the number of user demands $\sum_{i+1}^{N}\left|\mathbf{M}_{i}\right|$ (where $|$.$| denotes the cardinality of the set) plus the num-$ ber of different services supplied by the network.

Equilibrium Correspondence: The relation between the environment and a mechanism's equilibrium messages is represented by a point-to-set map, called equilibrium correspondence, and denoted by $\mu(: E \rightarrow \mathcal{M})$. The individual equilibrium correspondence of participant $i$, denoted by $\mu^{i}: E^{i} \rightarrow \mathcal{M}$, represents a relationship between the local environment of $i$ and the terminal messages emitted by $i$. 
To capture the private nature of the initial distribution of information, we require the following privacy-preserving property to be satisfied:

$$
\mu(e)=\cap_{i} \mu^{i}\left(e^{i}\right) .
$$

To determine the equilibrium correspondence for the first $N$ agents (the users) we define the Lagrangian function $\Lambda(\bar{x}, \lambda)$ (see [12]) of Problem P:

$$
\Lambda(\bar{x}, \lambda):=\sum_{i \in \mathbf{N}} \sum_{j \in \mathbf{M}_{i}} x_{j}^{i} r_{l, k}^{i, j}+\lambda_{l, k}\left(c_{l, k}-\sum_{i \in \mathbf{N}} \sum_{j \in \mathbf{M}_{i}} x_{j}^{i} r_{l, k}^{i, j}\right)
$$

with $\lambda_{l, k}$ being the Lagrangian multiplier corresponding to the $k^{\text {th }}$ resource on the $l^{\text {th }}$ link.

Using the first order optimality conditions, we define the equilibrium correspondence of the first $N$ agents (the users) as follows:

$$
\mu^{i}\left(U_{i}\left(\bar{x}_{i}\right)\right):=\left\{(\bar{p}, \bar{x}) \in \mathcal{M} \mid \frac{\partial}{\partial \bar{x}_{j}^{i}} U_{i}\left(\bar{x}_{i}\right)-p_{i, j} \leq 0 ; \bar{x}_{j}^{i}\left(\frac{\partial}{\partial \bar{x}_{j}^{i}} U_{i}\left(\bar{x}_{i}\right)-p_{i, j}\right)=0\right\}
$$

where $\bar{p}$ is the vector of prices for the services requested by the users, $\bar{x}$ is the vector of demands requested by the users, $\bar{x}_{i}$ is the vector of demands requested by user $i$ and $\bar{x}_{j}^{i}$ is the demand of user $i$ for service $j$.

From equation (13.12) and the Karush-Kuhn-Tucker (KKT) conditions, the equilibrium correspondence for agent $N+1$ (i.e. the network) is:

$$
\begin{gathered}
\mu^{N+1}\left(\mathbf{T}_{\mathbf{L}} \times \mathbf{R}_{\mathbf{T}_{\mathbf{L}}} \times \mathbf{K} \times c_{\mathbf{K}} \times \mathbb{F}_{i j}\left(\mathbf{R}_{\mathbf{T}_{\mathbf{L}}}, \mathbf{K}\right)\right) \\
:=\left\{(\bar{p}, \bar{x}) \in \mathcal{M} \mid \sum_{i \in \mathbf{N}} \sum_{j \in \mathbf{M}_{i}} x_{j}^{i} r_{l, k}^{i, j} \leq c_{l, k}, r^{i, j} \in \mathbb{F}_{i j}\left(\mathbf{R}_{\mathbf{T}_{\mathbf{L}}}, \mathbf{K}\right),\right. \\
\lambda_{l, k}\left(c_{l, k}-\sum_{i \in \mathbf{N}} \sum_{j \in \mathbf{M}_{i}} x_{j}^{i} r_{l, k}^{i, j}\right)=0, \\
\left.p_{i, j}=\sum_{l \in L, k \in K} r_{l, k}^{i, j} \lambda_{l, k}\right\} .
\end{gathered}
$$

Outcome Function: A function which translates messages into actions is called an outcome function, and it is denoted by $h(: \mathcal{M} \rightarrow \mathcal{A})$. In Problem $\mathbf{P}$ the outcome function is $h(\bar{p}, \bar{x}):=\bar{x}$.

\section{Key results}

The main features of the market mechanism of Section 13.3.1 are:

- The mechanism achieves the solution of the centralized resource allocation problem $\mathbf{P}$ and satisfies the informational constraints P.f and P.g. (see [125, 129, 132]). 
- The dimension of its message space is a lower bound on the dimension of the message space of any goal-realizing and regular mechanism for routing in unicast with QoS requirements. (See [125].)

- In the case of rate allocation with fixed routes and without QoS the mechanism is informationally efficient. ${ }^{9}$ (See $[125,128]$.)

\subsubsection{Multi-rate multicast}

\section{Problem formulation}

Let $\mathbf{N}=\{1,2, \ldots, N\}$ denote the set of users/agents requesting various services from a network. We assume that the network consists of a set of $L$ unidirectional links, with a topology denoted by $\mathbf{T}_{\mathbf{L}}$, and each link $l \in L$ having finite capacity $c_{l}$. There is a set $M$ of multicast groups. Each multicast group is a tree. Each multicast tree $m \in M$ is specified by $\left\{s_{m}, R_{m}, L_{m}\right\}$, where $s_{m}$ is the unique source node, $R_{m}$ is the set of receiver nodes, and $L_{m}$ is the set of links used by the group.

We denote by $R \triangleq \cup_{m \in M} R_{m}$ the set of all receivers over all the multicast groups, and by $R_{l, m}$ the set of all the receivers of multicast group $m \in M$ using link $l \in L$.

We assume that a unique user is connected to each receiver node $r \in R$. Each user $r$ has a utility function $U_{r}\left(x_{r}\right)$, where $x_{r}$ is the rate at which $r$ receives data. This utility function can be interpreted either in terms of the perceived quality of the service received or the amount paid in order to receive the service.

We make the following assumptions:

Assumption 1. The utility functions $U_{r}\left(x_{r}\right)$ are strictly concave, differentiable and increasing.

Assumption 2. The rates $x_{r}$ are assumed to be continuous variables.

Assumption 3. Rate allocations are done along fixed multicast trees with a fixed number of users.

Under the assumptions above, we consider the following network multi-rate multicast problem:

$$
\max _{x_{r}, r \in R} \sum_{r \in R} U_{r}\left(x_{r}\right)
$$

$\operatorname{Max} 1$

subject to:

$$
\begin{gathered}
\sum_{m \in M} \max _{r \in R_{l, m}} x_{r} \leq c_{l}, \quad \forall l \in L, \\
x_{r} \geq 0, \quad \forall r \in R,
\end{gathered}
$$

\footnotetext{
${ }^{9}$ Our notion of informational efficiency imposes more requirements on the properties of a mechanism than the standard notion of informational efficiency. To the best of our knowledge an analysis similar to that presented in $[125,128]$ was conducted only within the framework of production economies where the agent utility functions are of the Douglas Cobb form [88] or quadratic form [81].
} 
the informational constraints P.f and P.g of Section 13.3.1, and the following additional constraint:

Users are unaware of the method used for service delivery (e.g. unicast vs. multicast). P.h

Constraint Max 1.a is known as the capacity constraint. For this constraint to be satisfied, on each link, the sum of the rates used by each multicast tree can not exceed the link capacity. The capacity constraint ensures that for all the multicast trees, the rate on each branch of a tree is less than or equal to the rate on its parent branch.

Assumption P.h is critical in what follows, as it justifies the price-taking assumption we make in the sequel. If the method of service delivery is known to the users, then the mechanism proposed in Section 13.3.2 for the solution of problem Max 1 together with P.f-P.g may not be appropriate for multi-rate multicast service provisioning. This is because in this situation common links have features of public goods, and the mechanism proposed in Section 13.3.2 leads to the "free rider" problem [82, Chapter 11], which in the case of multi-rate multicast service provisioning manifests itself as follows: users who use a common link and demand less than the rate in the link do not participate in the price-sharing of the link (see $[125,126])$.

The multi-rate multicast problem with the features above is an informationally decentralized resource allocation problem where there are two distinct types of agents: network (network manager) and users. A major difference between the multi-rate multicast (under the assumptions above) and unicast is the fact that users connected to the same multicast tree receive service over "common links." Thus, to determine optimal (with respect to the performance criterion defined in Max 1) rate allocations in multi-rate multicast service provisioning one must find how the prices of "common links" should be shared by their users.

The goal in multi-rate multicast is to develop a mechanism for rate allocation along the various multicast trees in order to: (i) generate services that maximize a social welfare function consisting of the sum of individual user utility functions; and (ii) satisfy the informational constraints P.f-P.h.

Multicast service provisioning problems have received significant attention. Within the context of single rate and multi-rate multicast service provisioning, studies have addressed issues of bandwidth/rate allocation $[23,31,57,59,111,115-$ $119,122,134]$, routing $[27,28,102,121,144]$ and reliability $[30,34,60]$. Most of the literature on rate allocation is done via the notion of fairness $[23,31,111,115-$ 119, 122, 134], specifically, max-min fairness [14] and proportional fairness [63]. In particular, [119] develops a unified framework for diverse fairness objectives via the notion of fair allocation of utilities. A more general approach to rate allocation is via utility maximization. Utility maximizing is more general because rate allocation with the fairness property is utility maximizing when the utility has a special form [23, 86, 119, 122]. Although utility maximization has been extensively studied within the context of unicast rate allocation to achieve congestion control $[11,58,62,64,67,68,75,129,132]$, relatively fewer studies approached the multirate multicast allocation problem via a general utility maximization formulation, with 
the notable exceptions being [23, 57, 59]. Problem Max 1 together with Assumptions 13.3.2-13.3.2 and constraints Max 1.a, Max 1.b, P.f-P.h is similar in spirit to those formulated and analyzed in $[23,57,59]$. However, the decentralized resource allocation mechanism presented in this chapter is different from the mechanisms proposed in $[23,57,59]$. The development here follows $[125,126]$, where all the details of the proposed algorithm and of the key results can be found.

In the next section we present a market-based pricing mechanism that satisfies the informational constraints imposed by the nature of the network problem, and achieves a solution of the centralized optimization problem Max 1. This market mechanism is based on a price splitting algorithm and on properties of price splitting, all of which are presented formally in [126].

\section{Market mechanism}

We proceed as follows: We first describe a competitive market economy consisting of two types of agents: network and users. Then, within the context of this market we specify an iterative procedure (a tâtonnement process) which leads to an allocation that achieves a solution to Problem Max 1.

\section{Description of the market}

The market economy is composed of two types of agents: network (or network manager) and users. The network communicates directly with each user, and the users do not communicate with one another. The messages exchanged by the market agents are service prices and service demands.

For conceptual clarity we decompose the network manager into two distinct entities: service provider and auctioneer. The market features and the relations among the market agents are as follows: The resource traded at each link is the available communication rate. The rate price at link $l \in L$ is denoted by $\lambda_{l}$. The prices $\lambda_{l}$, $l \in L$, are set by the auctioneer. Based on $\lambda_{l}, l \in L$, the service provider sets up prices per unit of rate along each path of each multicast tree and communicates these prices to the users. Based on the service prices announced by the service provider the users demand a certain amount of service from the network in order to maximize their utility functions. Based on user demands the auctioneer updates the price per unit of rate at each link of the network.

We make the assumption that the service provider and users are price takers. They act as if their behavior has no effect on the equilibrium prices reached by the market allocation process. As pointed out in Section 13.3.2, this assumption is justified by P.h, that is, the fact that the users are unaware of the type of service received and they do not know the number of other users requesting service from the network. The price taking assumption and the fact that we try to maximize the users' utilities imply that: (i) the service provider will not attempt to make a profit; and (ii) the service prices are directly derived from resource prices.

Below we describe each type of agent in more detail. 
Service provider: The service provider receives from the auctioneer a rate price $\lambda_{l}$ for each link $l$ of the network. Based on these prices, it has to compute the price per unit of rate for each user.

A major challenge in solving multi-rate multicast problems through pricing is the determination of the set of user service prices from the set of link prices. This challenge comes from the fact that for each link which is common to multiple users of a multicast tree one needs to determine the portion of the price which is incurred by each of the users sharing the link. These price shares need to be determined in a way that satisfies the informational constraints imposed by the nature of the network multi-rate multicast problem.

In [126] we present a distributed algorithm which for a fixed set of link prices $\lambda$ computes a set of link price shares $\gamma(\lambda)$. Based on these price shares $\gamma(\lambda)$ the algorithm also computes the service prices $p(r, \lambda) \triangleq p(r, \gamma(\lambda))$ which generate demands that maximize the total user utility along any multicast tree for the fixed set of link prices $\lambda$.

Users: Users are price takers and request service from the service providers. For each user $r$ of the multicast tree $m \in M$ the service provider announces a service price $p(r, \lambda)$. Based on $p(r, \lambda)$, user $r$ determines its desired service rate by solving:

$$
x_{r}(p(r, \lambda)) \triangleq \underset{x}{\operatorname{argmax}}\left\{U_{r}(x)-p(r, \lambda) \times x\right\} .
$$

Auctioneer: The role of the auctioneer is to regulate the prices of resources, based on the aggregate excess demand vector $z(\lambda)$,

$$
z_{l}(\lambda) \triangleq \sum_{m \in M} \max _{r \in R_{l, m}} x_{r}(p(r, \lambda))-c_{l}
$$

at every link $l \in L$.

\section{The tâtonnement process}

We present a tâtonnmment process, described by an algorithm, called Algorithm 2, that describes how the market works. The algorithm proceeds iteratively as follows:

Step 1: The multicast trees are fixed.

Step 2: The auctioneer announces prices $\lambda:=\left\{\lambda_{l}, l \in L\right\}$ per unit of rate at each link of the network.

Step 3: The service provider receives the link prices $\lambda$ announced by the auctioneer. Given the link prices $\lambda$, the service provider communicates with the users via an iterative process in order to determine the optimal service prices $p(\lambda):=\left\{p_{i}(\lambda), i=1,2, \ldots, N\right\}$. During the iterative process the service provider and the users exchange prices per unit of service $p$ and service demands $x(p)$, with $x(p)$ satisfying (13.15). This iterative process is described in detail in [126, Section IV, Appendix A]. During the iterative process between 
the service provider and users, the auctioneer checks if the sign of the excess demand function $z(\lambda)$ is positive on some link or negative on all links.

Step 4: If at the end of Step $3 z(\lambda) \leq 0$ the process ends. Otherwise the auctioneer changes the prices $\lambda$ of resources according to a specific mechanism based on Scarf's algorithm (which is described in detail in $[125,126,132]$ ), announces new prices, say $\lambda^{\prime}$, and the process is repeated from Step 3 on.

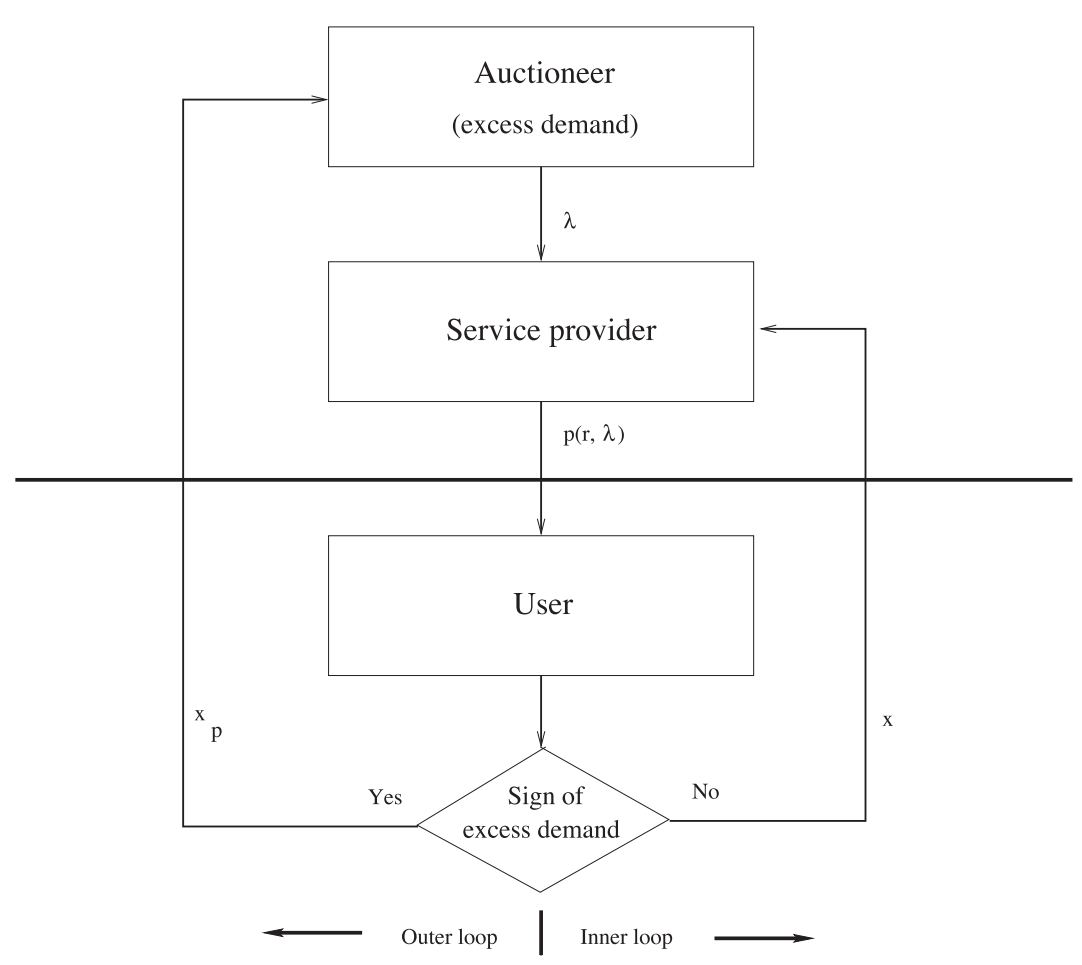

Fig. 13.2. Market mechanism.

The steps above are pictorially shown in Fig. 13.2. The figure illustrates the fact that the algorithm contains two loops: an outer loop and an inner loop. The inner loop describes the iterative process used by the service provider to determine user service prices $p(\lambda)$ (hence user demands) for fixed link prices $\lambda$ set by the auctioneer. For fixed $\lambda$ the inner loop also determines how prices of links that are common to many users are optimally shared by these users. The outer loop determines the iterative process used by the auctioneer to determine link prices based on excess demand. The iterative process of the inner loop is guided by the results of [126, Section III] 
and is presented in [126, Section IV, Appendix A]. The iterative process of the outer loop is guided by Scarf's Algorithm [120]. ${ }^{10}$

\section{Embedding into Hurwicz's framework}

We embed the multi-rate multicast problem, formulated in Section 13.3.2, within Hurwicz's abstract framework described in Section 13.2.2.

\section{The resource allocation problem:}

Environment: For the network Problem Max 1, the local environment $E^{i}$ of each user $i$ is the set of differentiable concave functions on $\mathbb{R}_{+}$. The local environment of the network is the set $E^{N+1} \triangleq\left\{\left\{\mathbf{T}_{\mathbf{L}}\right\} \times\{L\} \times\{M\} \times\left\{c_{L}\right\}\right\}$. The system environment is denoted by $E:=\left(\otimes_{i \in \mathbf{N}} E^{i}\right) \otimes E^{N+1}$.

Action Space: The action space is the feasible region of Problem Max $\mathbf{1 .}$

Goal Correspondence: For problem Max 1, $\pi: E \rightarrow \mathbf{A}$ is defined as follows:

$$
\pi(e):=\operatorname{argmax} \operatorname{Max} 1(e) .
$$

The environment, action space and goal correspondence describe the resource allocation problem.

\section{Mechanism specification}

Message Space: In the pricing mechanism proposed in Section 13.3.2 for solving Problem Max 1, two types of messages are exchanged among agents:

- $\quad$ To each user $i$ the network communicates a service price $p_{i}$.

- $\quad$ Each user $i$ communicates a service demand $x_{i}$ to the network.

Thus, the message space for Problem Max 1 has dimension equal to the number of user demands $\sum_{m \in M}\left|R_{m}\right|$ (where $|$.$| denotes the cardinality of the set) plus the$ number of service prices ${ }^{11}$.

Equilibrium Correspondence: Using the first order optimality conditions, we define the equilibrium message correspondence of the first $N$ agents (the users) as follows:

$$
\mu^{r}\left(U_{r}\left(x_{r}\right)\right):=\left\{\left(p_{r}, x_{r}\right) \in \mathcal{M} \mid \frac{\partial}{\partial x_{r}} U_{r}\left(x_{r}\right)-p_{r} \leq 0 ; x_{r}\left(\frac{\partial}{\partial x_{r}} U_{r}\left(x_{r}\right)-p_{r}\right)=0\right\} .
$$

\footnotetext{
${ }^{10}$ It may be possible to use algorithms other then Scarf's at the outer loop, however, to prove convergence of such algorithms we may need to impose additional constraints on the users' utility functions (e.g. second order differentiability of the utility functions).

${ }^{11}$ In this setup since no two services are identical (i.e. no two services are part of the same multicast tree and are delivered over the same links) the number of service prices is equal to the number of user demands.
} 
To present the equilibrium message correspondence for the network, we consider the following problem:

$$
\max _{x_{r}, r \in R} \sum_{r \in R} U_{r}\left(x_{r}\right)
$$

such that:

$$
\begin{gathered}
\sum_{m \in M} x_{r_{l, m}} \leq c_{l}, \quad \forall l \in L, \forall r_{l, m} \in R_{l, m} . \\
x_{r_{l, m} \geq 0,} \geq \quad \forall l \in L, \forall r_{l, m} \in R_{l, m}
\end{gathered}
$$

where $r_{l, m}$ denotes a receiver on the $\mathrm{m}^{\text {th }}$ multicast tree that employs link $l$.

Let $|M|$ denote the number of multicast trees in the network. We define the set $\Phi(l) \triangleq\left\{\left(r_{l, 1}, \ldots, r_{l,|M|}\right): r_{l, i} \in R_{l, i}, 1 \leq i \leq|M|\right\}$, to be the set of $|M|$ tuples, each tuple consisting of one receiver from each multicast tree, and every receiver of each tuple is downstream from link $l \in L$ on its respective multicast tree. We note that the number of elements in $\Phi(l)$ corresponds to the number of constraints for link $l$ in the set of equations (13.19). We denote by $r_{l}$ an element of $\Phi(l)$, and by $r_{l, m}$ a receiver on the $m^{t h}$ multicast tree of $r_{l}$. Note that if for some multicast tree $m \in M$ and some link $l \in L, R_{l, m}=\emptyset$, i.e. link $l$ is not part of the multicast tree $m$, then we let the $r_{l, m}$ entry of the $r_{l}$ tuple be empty, i.e. no receiver from multicast tree $m$ is assigned to any of the $r_{l}$ tuples. We define the set $\Phi(l, r) \triangleq\left\{\left(r_{l, 1}, \ldots, r_{l,|M|}\right): r \in\left\{r_{l, 1}, \ldots, r_{l,|M|}\right\}, r_{l, i} \in R_{l, i}, 1 \leq i \leq|M|\right\}$ to be a subset of $\Phi(l)$ where all the tuples contain receiver $r$.

Using the notation above we can rewrite equation (13.19) as follows:

$$
\sum_{m \in M} x_{r_{l, m}} \leq c_{l}, \quad \forall l \in L, \forall r_{l} \in \Phi(l) .
$$

Then, the Lagrangian function for Problem Max 2 can be expressed as:

$$
\Lambda(x, \gamma) \triangleq \sum_{r \in R} U_{r}\left(x_{r}\right)-\sum_{l \in L} \sum_{r_{l} \in \Phi(l)} \zeta_{r_{l}}\left(\sum_{m \in M} x_{r_{l, m}}-c_{l}\right)
$$

where $\zeta \triangleq\left\{\zeta_{r_{l}}: \zeta_{r_{l}} \in R_{+}, r_{l} \in \Phi(l), l \in L\right\}$.

Consequently, the first order optimality conditions are:

$$
\begin{gathered}
\zeta_{r_{l}}\left(\sum_{m \in M} x_{r_{l, m}}-c_{l}\right)=0 \\
\left(\frac{\partial U_{r}\left(x_{r}\right)}{\partial x_{r}}-\sum_{l \in \mathcal{L}_{r}} \sum_{r_{l} \in \Phi(l, r)} \zeta_{r_{l}}\right)=0
\end{gathered}
$$

where $\mathcal{L}_{r}$ is the set of links connecting receiver $r$ to the source.

From equations (13.22) and (13.23), the equilibrium correspondence for agent $N+1$ (i.e., the network) is: 


$$
\begin{gathered}
\mu^{N+1}\left(\left\{\mathbf{T}_{\mathbf{L}}\right\} \times\{L\} \times\{M\} \times\left\{c_{L}\right\}\right) \\
:=\left\{(\bar{p}, \bar{x}) \in \mathcal{M} \mid \sum_{m \in M} \max _{r \in R_{l, m}} x_{r} \leq c_{l}, \zeta_{r_{l}}\left(\sum_{m \in M} x_{r_{l, m}}-c_{l}\right)=0\right. \\
\left.p_{r}=\sum_{r_{l} \in \Phi(l, r)} \zeta_{r_{l}}\right\}
\end{gathered}
$$

where $\bar{p}:=\left(p_{1}, p_{2}, \ldots, p_{|R|}\right)$ is the vector of prices for the services requested by the users, and $\bar{x}:=\left(x_{1}, x_{2}, \ldots, x_{|R|}\right)$ is the vector of demands requested by the users.

Outcome Function: In Problem Max 1 the outcome function is $h(\bar{p}, \bar{x}):=\bar{x}$.

\section{Key results}

The main features of the market mechanism of Section 13.3.2 are:

- It achieves the solution of the centralized problem Max 1, and satisfies the informational constraints (P.f) and (P.g) (see [125, 126]).

- It is informationally efficient (see [125]).

\subsubsection{Discussion}

In Sections 13.3.1 and 13.3.2, we presented an approach for optimal resource allocation for both unicast with routing and QoS requirements, and multi-rate multicast service provisioning. The main features of this approach are:

(1) The objective to maximize the total value of the network to its users.

(2) The agents are price takers in the markets in which they participate.

(3) The users' utility functions $u_{i}$ are quasi-linear, continuously differentiable, and strictly concave.

(4) There is no cost associated with the supply of network resources.

We now briefly discuss and critique each one of the features above separately. For more details we refer the reader to [125, 126, 129, 132].

(1) In the problems considered we assumed that the objective function of interest was to maximize the sum of individual network users' utility functions. It may not be obvious why this is a reasonable objective to consider.

It is important to realize that our point of view is primarily normative, not descriptive. That is, we have taken a particular objective function-one which we believe is often reasonable_-and studied whether a network resource pricing scheme exists that can achieve an optimum for that particular function, and how one might implement that allocation with a market-based algorithm. Thus, we have demonstrated the feasibility of using pricing to achieve a particular performance goal. We are not claiming that this goal describes any particular actual network environment. Nor are we making the stronger normative claim that this objective function should be adopted in any particular setting. 
We do, in fact, believe that maximizing the sum of user utilities is a reasonable description for a wide variety of network allocation problems. Suppose we are considering a corporate intranet. If the corporation's overall objective is to maximize its profits (in present value), then the appropriate interpretation of our problem is to define each user's "utility" as that user's contribution to corporate profit as a function of the network services it consumes. In other words, the corporation is not (directly) interested in how personally happy an employee is with the network, but on how much the network enhances the employee's productivity. Then the sum of user utilities will be the contribution of network services to corporate profits, which is precisely the firm's objective function for this part of the overall management problem. Although it may seem difficult to come up with a reasonable representation of the effect of network services on each user's contribution to corporate profits, at some level this is precisely the problem corporations need to solve for allocating equipment, office space, subordinates and so forth to each employee-it is well beyond the scope of our research to worry about how the corporation specifically formulates these valuation functions.

Thus, although our method of using prices to allocate network resources cannot be directly applied to every allocation problem with any reasonable objective function, we believe that it has broad applicability to many existing situations. In any case, when our objective function $i s$ the desired goal, we have carefully analyzed the existence and implementability of a pricing scheme to support that objective.

(2) For the problems presented in Sections 13.3.1 and 13.3.2, we have imposed the price-taking assumption to the agents of the market economy. How useful is the price-taking assumption? It is not essential for a proof that an algorithm exists that will clear the markets and reach some equilibrium allocation of network resources. However, in general, that allocation will not be a solution of our original optimization problem.

As a general matter we could show that equilibrium allocations based on behavior other than price-taking will lead to less efficient allocations, that is, allocations that do not maximize the sum of user utilities subject to the technology constraints. Therefore, we did not consider markets in which agents exhibit different types of strategic behavior, but limited ourselves to the price-taking behavior that we can show can be harnessed to yield a solution to the centralized optimization problem.

Restricting attention to the price-taking case may not in practice be as restrictive as it seems. Consider the example of a corporate intranet with a single monopoly provider of resources and services. If the management instructs the resource and service provider to behave "as if" it is a price taker (and provides compensation incentives that make it in the provider's best interests to do so) then the desired outcome can be achieved. Essentially, this requires compensating the provider based on the value of the allocation to the company as a whole, rather than based on the provider's own local "profits." If the network is to be managed with an agent-based control system, the agents should be programmed to act as price takers, whether or not other programmable strategies might seem more desirable from the local viewpoint of the agents. 
In a more open, conventionally market-based system, such as a commercial market for virtual data circuits, it is also possible that at a given moment some participants might have some market power, which is to say that they are cognizant of an opportunity to improve their position by acting strategically with respect to pricesetting, rather than as a price taker. In such a setting, it might not be possible to directly control behavior to make those participants behave "as if" they are price takers. However, if there are no artificial barriers to entry by other providers-for example, if it is possible for another competing firm to build an interconnected network of links with buffers and bandwidth - then it will tend to be the case that in a long-run equilibrium surviving agents will be those who behave as price takers (competition will drive others out of the market). Therefore, we believe there are many circumstances under which the conditions will exist, or can be imposed, that are necessary for our approach to provide an equilibrium that is a social welfare maximizing solution of the centralized network problem.

The price-taking assumption is harder to justify in multi-rate multicast service provisioning than in unicast with routing. Treating users as price-takers in Problem Max 1 is reasonable under the assumption that they are unaware of the method of service delivery (assumption P.h). Without such an assumption, a formulation of multi-rate multicast as a public goods problem, albeit a non-typical one, may be more appropriate than that of Section 13.3.2. The investigation of Problem Max 1 without assumption P.h remains an interesting open problem.

(3) Since we assume that the expenditure of the good under study is a small portion of a consumer's total expenditure, the small size of the market under study should lead the prices of the other goods to be approximately unaffected by changes in this market. Because of this fixity of other prices, we are justified in treating the expenditure on these other goods as a single composite commodity, which we call the numeraire. This allows us to express the utility function as a function of the goods under study and the numeraire.

The choice of representing users' preferences by quasi-linear objective functions also imposes the constraint that there are no income effects on network service demand; that is, changes in income or budget available to the users does not change the amount of network services they wish to purchase. This is a typical simplifying assumption in the economic literature when the budget share of the services of interest is small, e.g. when network services are only a relatively small amount of the users' total expenditures.

The rest of the assumptions made for the utility functions are normal assumptions usually made in analysis of economic optimization problems. The continuously differentiable assumption comes from the idea that we may look at a set of users that may have similar utilities as a group, and in this case the group utility will be a smoothed out version of each user's utility. Strictly concave assumption is natural when we are working with goods that are desirable.

(4) In both network problems considered in Sections 13.3.1 and 13.3.2, we assumed that there is no cost in supplying network resources (bandwidth, buffers, etc.) to the market. This cost can be incorporated into our model if we subtract it from the 
objective function of the optimization problem. We believe that the new problem will have the same qualitative properties with the problem presented in this chapter, thus it may lead to a similar type of result.

\subsection{Mechanism Design in Networks: An Implementation Theory Point of View}

In the previous section we considered a mechanism to be a set of rules which, if followed, generate allocations that satisfy a goal correspondence. This kind of mechanisms ignore issues of strategic behavior of individual agents. Thus, it may not be possible to contractually enforce such mechanisms. To design resource allocation mechanisms that are contractually enforceable we have to take into account the divergence of individual preferences from the overall performance objective.

In this section we discuss game forms that implement social choice rules characterized by socially welfare maximizing solutions. We concentrate on Nash implementation and relate our discussion to the unicast problem with routing.

There are two distinct ways in which one can think of implementation of social welfare maximization rules in Nash equilibria. We present them below.

When Nash implementation is the solution concept, an individual (user) needs to know not only his own preferences, but everyone else's preferences so as to determine his equilibrium message(s). Thus, for Nash implementation purposes in unicast and routing, an environment of a user is an entire profile of utility functions and $\left\{\left\{\mathbf{T}_{\mathbf{L}}\right\} \otimes\left\{\mathbf{R}_{\mathbf{T}_{\mathbf{L}}}\right\} \otimes\{\mathbf{K}\} \otimes \mathbb{F}_{i j}\left(\mathbf{R}_{\mathbf{T}_{\mathbf{L}}}, \mathbf{K}\right) \otimes\left\{c_{\mathbf{K}}\right\}\right\}$, defined in Section 13.3.2. Consequently, the space of user's environments is

$$
\hat{E}:=\left(\otimes_{i \in \mathbf{N}} E^{i}\right) \otimes\left\{\left\{\mathbf{T}_{\mathbf{L}}\right\} \otimes\left\{\mathbf{R}_{\mathbf{T}_{\mathbf{L}}}\right\} \otimes\{\mathbf{K}\} \otimes \mathbb{F}_{i j}\left(\mathbf{R}_{\mathbf{T}_{\mathbf{L}}}, \mathbf{K}\right) \otimes\left\{c_{\mathbf{K}}\right\}\right\},
$$

where all the components of the right-hand-side of (13.25) are defined in Section 13.3.2. When the action space $\mathbf{A}$ is the feasible region of Problem $\mathbf{P}$, and the message space $\mathcal{M}$ is

$$
\mathcal{M}=\hat{E} \times \mathbf{A} \times \mathbb{N}
$$

where $\mathbb{N}$ is the set of natural numbers, then the goal correspondence $\pi: \otimes_{i=1}^{N} E^{i} \rightarrow$ $\mathbf{A}$, described by the centralized solution of Problem $\mathbf{P}$, can be implemented in Nash equilibria by a game form $(\mathcal{M}, h)$ where the outcome function $h$ is defined in [84, Theorem 3]. Such an implementation is possible for the following reason. For the unicast problem with routing, $\pi$ is a Pareto correspondence; Pareto correspondences are monotonic and possess the no veto power property [83, 84]; therefore, $\pi$ can be implemented in Nash equilibria by the aforementioned game form whenever the number of users in the network is greater than or equal to three [83, 84]. However, the game form described above is infinite dimensional. Thus, the approach above to Nash implementation leads to game forms that are infeasible on information grounds. 
An alternative way of proceeding with Nash implementation of the goal correspondence $\pi$ (which could potentially result in game forms with finite dimensional message spaces) is the following. Consider that users know their own environment, but not those of other users or the network. All users are involved in an unspecified message exchange process in which they grope their way to a stationary message and in which the Nash property is a necessary condition for stationarity. Experimental evidence [124] has shown that such an approach to Nash implementation is reasonable.

An important open issue within the context of the second approach to Nash implementation is: What is the minimum dimensionality of the message space of game forms that implement social welfare maximizing rules (e.g. $\pi$ ) in Nash equilibria? It is expected that, in general, an implementing mechanism with the Nash property in equilibrium messages will require a larger message space than the one that suffices for decentralized realization without regard to individual incentives. Reichelstein and Reiter [107] have shown that the statement above is true in the case of Nash implementation of Walrasian allocations in exchange environments. The following example from [107] illustrates the fact that Nash implementations require larger message spaces than the corresponding decentralized realizations.

\section{Example 4.1}

Consider a resource allocation problem with two agents $\{1,2\}$ and two goods $\{X, Y\}$, where good $X$ represents a desirable service and good $Y$ has the interpretation of money. Assume that the agents' preference over the goods are described, at least locally, by quasi-linear ${ }^{12}$ convex utility functions of the form

$$
U_{i}\left(x, y \mid e_{i}\right) \triangleq e_{i} \times x-\frac{x^{2}}{2}+y, \quad e_{i} \in E_{i}, i \in\{1,2\} .
$$

The agents' private objective is to maximize their individual utility function, while the social objective is to achieve a resource allocation which maximizes the sum of individual utility functions.

Assume that the goods are distributed among the agents and the agents are permitted to trade. From the realization theory point of view there exists a goal realizing mechanism with a message space of dimension two [43]. Specifically, in the case in which both agents are truthful, the mechanism in which agent 1 sets the price for good $X$ and agent 2 makes a request ${ }^{13}$ for good $X$ based on the price set by agent 1 is social welfare maximizing.

In [107] the authors show that there is no mechanism of dimension 2 which implements in Nash equilibria the social welfare maximizing rule for this problem. In particular, they show that the pricing mechanism where agent 1 sets the price

${ }^{12}$ Let $X:=\left\{x_{1}, x_{2}, \ldots, x_{L}\right\}$ be a set of commodities. A function $U(x)$ is called quasilinear with respect to commodity $L$ if it is of the form $U(x)=\mathcal{U}\left(x_{1}, x_{2}, \ldots, x_{L-1}\right)+x_{L}$. Commodity $x_{L}$ is called the numeraire commodity. The numeraire commodity generally has the interpretation of money.

${ }^{13}$ If agent 2 makes a negative request for good $X$ it means that he would like to sell that amount of good $X$ to agent 2 . 
for commodity $X$ and agent 2 makes a request for this commodity based on the price, does not have a Nash equilibrium which is social welfare maximizing. The authors present a mechanism of dimension 3 which implements the social welfare maximizing rule in Nash equilibria. This mechanism works as follows:

The agents message spaces are described by

$$
\begin{aligned}
& M_{1}=\left\{m_{1} \mid m_{1} \in \mathbb{R}_{+}\right\}, \\
& M_{2}=\left\{\left(m_{2}, m_{3}\right) \mid\left(m_{2}, m_{3}\right) \in \mathbb{R}_{+}^{2}\right\} .
\end{aligned}
$$

The amount of commodity $X$ exchanged is characterized by the outcome functions

$$
\begin{aligned}
& h_{1}^{x}(m)=m_{1}-m_{2}, \\
& h_{2}^{x}(m)=m_{2}-m_{1},
\end{aligned}
$$

while the amount of the numeraire commodity $Y$ exchanged by the agents is described by:

$$
\begin{aligned}
& h_{1}^{y}(m)=-m_{3}\left(m_{1}-m_{2}\right), \\
& h_{2}^{y}(m)=-m_{1}\left(m_{2}-m_{1}\right)-\left(m_{1}-m_{3}\right)^{2} .
\end{aligned}
$$

In this mechanism $m_{3}$ has the interpretation of the price of good $X$. Agent 1 maximizes his utility function based on $m_{3}$ and sends his message $m_{1}$ to agent 2 . Agent 2 does not use $m_{3}$ as the price for commodity $X$, but rather it uses message $m_{1}$. Based on $m_{1}$, agent 2 maximizes his utility by choosing $m_{2}$. Agent 2 receives a quadratic penalty for announcing a price which is not equal to $m_{1}$. This penalty, along with the fact that he does not set his own price for good $X$, forces agent 2 to be truthful in his messages. In [107] the authors prove that this mechanism implements the social welfare maximizing rule in Nash equilibria.

Recent game theoretic studies in network unicast problems (without routing)[35, 50-52, 114] have shown that: When the dimension of the message space of the game form is the same as that of the pricing mechanism which suffices for decentralized realization, the game form does not implement the social welfare maximizing rule. Specifically the Nash equilibria determined in [35, 50-52, 114] are not social welfare maximizing.

Example 4.1, the results in [35, 50-52, 114], as well as the results on implementation in other solution concepts such as Bayesian Nash equilibria [98], and refinements of Nash equilibria (specifically, subgame perfect equilibria [87], and undominated Nash equilibria [18]) reveal that:

I1: Games that are induced by game forms whose message space has the same dimension as that of the standard pricing mechanism have multiple equilibria, some of which do not result in welfare maximizing solutions. Consequently, such game forms can not implement (in the corresponding solution concept) social welfare maximizing rules. 
I2: Implementation of social welfare maximizing rules in some solution concept (cf. Section 13.2.3) requires a message space the dimension of which is larger than that of the message space which suffices for decentralized realization.

I3: The increase in the dimension of the message space must accomplish the following:

1. It must eliminate the equilibria that do not result in welfare maximizing allocations (cf. I1).

2. It must maintain the equilibria that result in welfare maximizing allocations.

3. If must not introduce additional equilibria, unless these equilibria result in welfare maximizing allocations.

4. It must induce price-taking behavior among the players (users).

We have the following conjecture concerning the Nash implementation of the centralized solution of the unicast network resource allocation problem with routing and QoS requirements.

Conjecture: In the case of unicast service provisioning, with $N$ users and $L$ services, there exists a game form which implements the centralized solution of Problem $\mathbf{P}$ in Nash equilibria and has a message space of dimension equal to the dimension of the pricing mechanism plus $\left\lceil\frac{L}{N}\right\rceil$. Any mechanism with a message space of smaller dimension can not implement the centralized solution of Problem $\mathbf{P}$ in Nash equilibria [127].

\subsection{Conclusion}

Our goal was to discuss: (i) how decentralized network resource allocation problems fit within the context of mechanism design; and (ii) how mechanism design can provide guidelines for the determination of resource allocation strategies that realize (in an informationally efficient manner) social welfare maximizing resource allocation rules, and implement them in some appropriate behavioral equilibrium concept (e.g. Nash equilibrium) in an informationally efficient manner. Our discussion was guided by two classes of network resource allocation problems (unicast with routing and QoS requirements, and multi-rate multicast) that received significant attention in the engineering world. The results we presented reveal the connection between network resource allocation and mechanism design. The discussion also revealed that: (1) the aforementioned network problems are better understood from the realization theory point of view than from the implementation theory viewpoint. (2) A formulation that is appropriate for the multi-rate multicast problem when the users are aware of the method of service delivery remains an interesting open problem.

In our opinion, two problems of fundamental importance are: (1) the characterization and classification of mechanisms in terms of their "communication" and "information processing" requirements; and (2) how can the theory of implementation guide the design of minimal message space mechanisms that implement, in some appropriate solution concept, social welfare maximizing network resource allocation rules. 
Acknowledgment. This research was supported in part by ONR Grant N0001403-1-0232. The authors gratefully acknowledge stimulating discussions with Jeffrey MacKie-Mason and John Ledyard.

\section{References}

[1] D. Abreu and H. Matsushima, Virtual implementation in iteratively undominated strategies: Complete information, Econometrica, 60 (5):993-1008, 1992.

[2] D. Abreu and A. Sen, Subgame perfect implementation: A necessary and almost sufficient condition, Journal of Economic Theory, 50:285-299, 1990.

[3] M. Allais, A la Recherche d'une Discipline Economique, I, Paris, 1943.

[4] M. Allais, Economie Pure et Rendement Social, Paris, 1945.

[5] T. Apostol, Mathematical Analysis, Addison-Wesley, 1974.

[6] K. Arrow, A difficulty in the concept of social welfare, Journal of Political Economy, 58:328-346, 1950.

[7] K. Arrow, An extension of the basic theorems of welfare economics, In J. Neyman, Ed., Proceedings of the Second Berkeley Symposium, 507-532, Berkeley, CA, 1951.

[8] K. Arrow, Social Choice and Individual Values, Yale University Press, 1951.

[9] K. Arrow and F. Hann, General Competitive Analysis, Holden-Day, San Francisco, 1971.

[10] E. Barone, Il ministro della produzione nello stato collettivista, Giornale degli Economisti, 1908.

[11] Y. Bartal, J. Byers and D. Raz, Global optimization using local information with applications to flow control, In Proceedings of the $38^{\text {th }}$ Ann. IEEE Symp. on Fundations of Computer Science (FOCS), Miami, FL, October 1997.

[12] M. Bazaraa, H. Sherali and C. Shetty, Nonlinear Programming Theory and Algorithms, John Wiley, New York, 1993.

[13] A. Bergson, A reformulation of certain aspects of welfare economics, Quarterly Journal of Economics, 52:310-334, 1938.

[14] D. Bertsekas and R. Gallagher, Data Networks, 2nd Edition, Prentice Hall, Englewood Cliffs, 1992.

[15] N. Bukharin, Economics of the Transition Period, 1920.

[16] N. Bukharin and E. Preobrazhensky, The ABC of Communism, Penguin Books, 1969.

[17] R. Cocchi, D. Estrin, S. Shenker and L. Zhang, Pricing in computer networks: motivation, formulation and example, IEEE/ACM Transaction on Networking, 1 (6):614-627, December 1993.

[18] L. Corchon, The Theory of Implementation of Socially Optimal Decisions in Economics, McMillan, New York, 1996.

[19] C. Courcoubetis, F. Kelly and R. Weber, Measurement-based usage charges in communications networks, Operations Research, 48 (4):535-548, 2000. 
[20] P. Dasgupta, P. Hammond and E. Maskin, The implementation of social choice rules: Some general results on incentive compatibility, Review of Economic Studies, 46:185-216, 1979.

[21] T. de Scitovsky, A note on welfare propositions in economics, Review of Economic Studies, 9:77-88, 1941.

[22] G. de Veciana and R. Baldick, Resource allocation in multiservice networks via pricing, Computer Networks and ISDN Systems, 30:951-962, 1998.

[23] S. Deb and R. Srikant, Congestion control for fair resource allocation in networks with multicast flows, IEEE/ACM Transactions on Networking, 12 (2):261-273, 2004.

[24] G. Debreu, Coefficient of resource utilization, Econometrica, 19:273-292, 1951.

[25] G. Debreu, Theory of Value, John Wiley, New York, 1959.

[26] H. Dickinson, Price formation in a socialist community, The Economic Journal, 237-250, 1933.

[27] M. Donahoo, K. Calvert and E. Zegura, Center selection and migration for wide-area multicast routing, Journal of High Speed Networks, 6 (2), 1997.

[28] M. Donahoo and E. Zegura, Core migration for dynamic multicast routing, In International Conference on Computer Communications and Networks (ICCCN), Washington, DC, 1996.

[29] M. Doob, Economic theory and the problems of a socialist economy, The Economic Journal, 588-598, 1933.

[30] N. Duffield, J. Horowitz, D. Towsley, W. Wei and T. Friedman, Multicastbased loss inference with missing data, IEEE Journal on Selected Areas in Communications, 2002.

[31] E. Graves, R. Srikant and D. Towsley, Decentralized computation of weighted max-min fair bandwidth allocation in networks with multicast flows, In Proceedings Tyrrhenian International Workshop on Digital Communications (IWDC), Taormina, Italy, 2001.

[32] J. Green and J. Laffont, Incentives in Public Decision Making, North-Holland, Amsterdam, 1979.

[33] A. Gupta, D. Stahl and A. Whinston, A stochastic equilibrium model of internet pricing, Journal of Economic Dynamics and Control, 21:697-672, 1997.

[34] R. Gupta and J. Walrand, Average bandwidth and delay for reliable multicast, In E. Gelenbe, Ed., System Performance Evaluation - Methodologies and Applications, chapter 13, 191-202, CRC Press, 2000.

[35] B. Hajek and S. Yang, Strategic buyers in a sum bid game for flat networks, 2004, preprint.

[36] J. Hicks, The foundations of welfare economics, Economic Journal, 69:696$712,1939$.

[37] H. Hotelling, Edgeworth's taxation paradox and the nature of supply and demand functions, Journal of Political Economy, 40:577-616, 1932.

[38] H. Hotelling, The general welfare in relation to problems of taxation and of railway and utility rates, Econometrica, 6 (3):242-269, 1938. 
[39] L. Hurwicz, Optimality and informational efficiency in resource allocation processes, In K. Arrow, S. Karlin and P. Suppes, Eds., Mathematical Methods in the Social Sciences, Stanford University Press, 1960.

[40] L. Hurwicz, On informationally decentralized systems, In B. McGuire and R. Radner, Eds., Decision and Organization, Volume in Honor of Jacob Marschak, 297 - 336, North Holland, 1972.

[41] L. Hurwicz, The design of mechanisms for resource allocation, American Economic Review, 63 (2):1-30, 1973.

[42] L. Hurwicz, On the dimensional requirements of informationally decentralized Pareto satisfactory processes, Studies in Resource Allocation Processes, 1977.

[43] L. Hurwicz, On informational decentralization and efficiency in resource allocation mechanisms, In S. Reiter, Ed., MAA Studies in Mathematical Economics, 25:238-350, Mathematical Association of America, 1986.

[44] L. Hurwicz, S. Reiter and D. Saari, On constructing mechanisms with message spaces of minimal dimension for smooth performance functions, mimeo, Northwestern University, 1985.

[45] M. Jackson, Bayesian implementation, Econometrica, 59:461-478, 1991.

[46] M. Jackson, Implementation of undominated strategies, Review of Economic Studies, 59:757-775, 1992.

[47] M. Jackson, A crash course in implementation theory, Social Choice and Welfare, 18:655-708, 2001.

[48] M. Jackson, T. Palfrey and S. Srivastava, Undominated Nash implementation in bounded mechanisms, Games and Economic Behavior, 6:474-501, 1994.

[49] H. Jiang and S. Jordan, The role of price in the connection establishment process, European Transactions on Telecommunications, 6 (4):421-429, JulyAug. 1995.

[50] R. Johari, S. Mannor, and J. Tsitsiklis, Efficiency loss in a network resource allocation game: the case of elastic supply, 2004, preprint.

[51] R. Johari and J. Tsitsiklis, Efficiency loss in a network resource allocation game, Mathematics of Operations Research, 29 (3):407-435, 2003.

[52] R. Johari and J. Tsitsiklis, Efficiency loss in a Cournot mechanism for network resource allocation, 2004, preprint.

[53] J. Jordan, The informational requirements of local stability in decentralized allocation mechanisms, In T. Groves, R. Radner and S. Reiter, Eds., Information, incentives and economic mechanisms: essays in honor of Leonid Hurwicz, 183-212, University of Minnesota Press, 1987.

[54] S. Jordan and H. Jiang, Connection establishment in high speed networks, IEEE Selected Areas in Communications, 13 (7):1150-1161, 1995.

[55] N. Kaldor, Welfare propositions in economics and inter-personal comparisons of utility, Economic Journal, 49:549-552, 1939.

[56] H. Kanemitsu, Informational efficiency and decentralization in optimal resource allocation, The Economic Studies Quarterly, 16:22-40, 1966.

[57] K. Kar, S. Sarkar and L. Tassiulas, Optimization based rate control for multirate multicast sessions, In Proceedings of INFOCOM, Alaska, 2001. 
[58] K. Kar, S. Sarkar and L. Tassiulas, A simple rate control algorithm for maximizing total user utility, In Proceedings of INFOCOM, Alaska, 2001.

[59] K. Kar, S. Sarkar and L. Tassiulas, A scalable low overhead rate control algorithm for multirate multicast sessions, IEEE Journal of Selected areas in Communication, Special Issue in Network Support for Multicast Communications, 20 (8):1541-1557, October 2002.

[60] S. Kasera, G. Hjalmtysson, D. Towsley and J. Kurose, Scalable reliable multicast using multiple multicast channels, IEEE/ACM Transactions on Networking, 8 (3):294-310, 2000.

[61] K. Kautsky, The Social Revolution, Charles Kerr \& Co., 1903.

[62] F. Kelly, On tariffs, policing and admission control for multiservice networks, Operations Research Letters, 15:1-9, 1994.

[63] F. Kelly, Charging and rate control for elastic traffic, European Transactions on Telecommunication, 8 (1):33-37, 1997.

[64] F. Kelly, A. Maulloo, and D. Tan, Rate control for communication networks: shadow prices, proportional fairness and stability, Operational Research Society, 49:237-252, 1998.

[65] T. Koopmans, Analysis of production as an efficient combination of activities, In T. Koopmans, Ed., Activity Analysis of Production and Allocation, (13):3337, Cowles Commission Monograph, New York, 1951.

[66] T. Koopmans, Three Essays on the State of Economic Science, McGraw-Hill, New York, 1957.

[67] S. Kunniyur and R. Srikant, End to end congestion control schemes: Utility functions, random losses and ECN marks, In Proceedings of INFOCOM, Tel Aviv, Israel, 2000.

[68] R. La and V. Anantharam, Charge-sensitive TCP and rate control on the internet, In Proceedings of INFOCOM, Tel Aviv, Israel, 2000.

[69] S. Lang, Linear Algebra, Springer-Verlag, New York, 1991.

[70] O. Lange, On the Economic Theory of Socialism, University of Minnesota Press, Minneapolis, 1938.

[71] O. Lange, The foundations of welfare economics, Econometrica, 10:215-228, 1942.

[72] V. Lenin, State and Revolution, International Publishers, 1918.

[73] A. Lerner, The concept of monopoly and the measurement of monopoly power, The Review of Economic Studies, 1:157-175, 1934.

[74] A. Lerner, Economic theory and socialist economy, The Review of Economic Studies, 2 (1):51-61, 1934.

[75] S. Low and D. Lapsley, Optimization flow control I: Basic algorithm and convergence, IEEE/ACM Transactions on Networking, 7 (6):861-874, 1999.

[76] S. Low and P. Varaiya, A new approach to service provisioning in ATM networks, IEEE/ACM Transactions on Networking, 1:547-553, 1993. see also [77] for corrections.

[77] S. Low and P. Varaiya, Corrections to: A new approach to service provisioning in ATM networks, IEEE/ACM Transactions on Networking, 2:312, 1994. 
[78] J. MacKie-Mason and H. Varian, Pricing congestible network resources, Journal of Selected Areas in Communications, 13 (7):1141-1149, 1995.

[79] J. MacKie-Mason and H. Varian, Some FAQs about usage-based pricing, Computer Networks and ISDN Systems, 28:257-265, 1995.

[80] J. Marschak and E. Lederer, Der neue mittelstand, Grundriss der Nationalokonomik, 1926.

[81] T. Marschak and S. Reichelstein, Network mechanisms, informational efficiency, and hierarchies, Journal of Economic Theory, 78:106-141, 1998.

[82] A. Mas-Colell, M. Whinston, and J. Green, Microeconomic Theory, Oxford University Press, New York, 1995.

[83] E. Maskin, The theory of implementation in Nash equilibrium: A survey, In L. Hurwicz, D. Schmeidler, and H. Sonnenschein, Eds., Social Goals and Social Organization, Chapter 6, 173-204, Cambridge University Press, 1985.

[84] E. Maskin, Nash implementation and welfare optimality, Review of Economic Studies, 66:23-38, 1999.

[85] E. Maskin and T. Sjőstrőm, Implementation theory, In K. Arrow, A. Sen and K. Suzumura, Eds., Handbook of Social Choice and Welfare, 1, chapter 5, North Holland, 2002.

[86] L. Massoulie and J. Roberts, Bandwidth sharing: Objectives and algorithms, In Proceedings of INFOCOM, New York, USA, 1999.

[87] J. Moore and R. Repullo, Nash implementation: A full characterization, Econometrica, 58 (5):1083-1099, 1990.

[88] K. Mount and S. Reiter, The informational size of message spaces, Journal of Economic Theory, 8:161-192, 1974.

[89] K. Mount and S. Reiter, Economic environments for which there are pareto satisfactory mechanisms, Econometrica, 45 (4):821-842, 1977.

[90] K. Mount and S. Reiter, On the existence of a locally stable dynamic process with a statically minimal message space, In T. Groves, R. Radner and S. Reiter, Eds., Information, Incentives and Economic Mechanisms: Essays in Honor of Leonid Hurwicz, 213-242, University of Minnesota Press, Minneapolis, 1987.

[91] L. Murphy and J. Murphy, Bandwidth allocation by pricing in ATM networks, In Proceedings of the IFIP Broadband Communications, 333-351, 1994.

[92] L. Murphy, J. Murphy and E. Posner, Distributed pricing for embedded ATM networks, In Proceedings of the International Teletraffic Congress, ITC-14, 1994.

[93] O. Neurath, Durch die Kriegswirtschaft zur Naturalwirtschaft, Callwey, München, 1919.

[94] T. Palfrey, Implementation theory, In R. Aumann and S. Hart, Eds., Handbook of Game Theory, III:2271-2326, Elsevier Science, 2002.

[95] T. Palfrey and S. Srivastava, Implementation with incomplete information in exchange economies, Econometrica, 57:115-134, 1989.

[96] T. Palfrey and S. Srivastava, Nash implementation using undominated strategies, Econometrica, 59:479-502, 1991. 
[97] T. Palfrey and S. Srivastava, Implementation in bayesian equilibrium: The multiple equilibrium problem in mechanism design, Volume I, In J. Laffont, Ed., Advances in Economic Theory, in Econometric Society Monographs, (20):283-323, Cambridge University Press, 1992.

[98] T. Palfrey and S. Srivastava, Bayesian Implementation, Fundamentals of Pure and Applied Economics, 53, Harwood academic, 1993.

[99] V. Pareto, La courbe des revenus, Le Monde economique, 1896.

[100] V. Pareto, The new theories of economics, Journal of Political Economics, 5:485-502, 1896.

[101] V. Pareto, Manual of Political Economy, Augustus Kelly, New York, 1906.

[102] W. Park, H. Owen and E. Zegura, Sonet/SDH multicast routing algorithms in symmetrical three-stage networks, In IEEE International Conference on Communications, Seattle, WA, 1995.

[103] C. Parris and D. Ferrari, A resource based pricing policy for real-time channels in a packet-switching network, Technical Report TR-92-018, International Computer Science Institute, Berkeley, CA, 1992.

[104] C. Parris, S. Keshav and D. Ferrari, A framework for the study of pricing in integrated networks, Technical Report TR-92-016, International Computer Science Institute, Berkeley, CA, 1992.

[105] N. Pierson, Das Wertproblem in der sozialistischen Gesellschaft, 1902.

[106] A. Postlewaite and D. Schmeideler, Implementation in differential information economies, Journal of Economic Theory, 39:14-33, 1986.

[107] S. Reichelstein and S. Reiter, Game forms with minimal message spaces, Econometrica, 56 (3):661-692, 1988.

[108] S. Reiter, Informational efficiency of iterative processes and the size of message spaces, Discussion Paper 11, Center for Mathematical Studies in Economics and Management Science, Northwestern University, 1972.

[109] S. Reiter, The knowledge revealed by an allocation process and the informational size of the message space, Discussion Paper 6, Center for Mathematical Studies in Economics and Management Science, Northwestern University, 1972.

[110] S. Reiter, Information and performance in the (new) welfare economics, The American Economic Review, 67 (1):226-234, 1977, Papers and Proceedings of the Eighty-ninth Annual Meeting of the American Economic Association.

[111] D. Rubenstein, J. Kurose and D. Towsley, The impact of multicast layering on network fairness, In Proceedings of ACM SIGCOMM, Cambridge, MA, 1999.

[112] T. Saijo, Strategy space reduction in Maskin's theorem: Sufficient conditions for Nash implementation, Econometrica, 56:693-700, 1988.

[113] P. Samuelson, Foundations of Economic Analysis, Harvard University Press, Cambridge, MA, 1947.

[114] S. Sanghavi and B. Hajek, Optimal allocation of a divisible good to strategic buyers, 2004, preprint.

[115] S. Sarkar and L. Tassiulas, Fair allocation of resources in multirate multicast trees, In Proceedings of Globecom, 1999. 
[116] S. Sarkar and L. Tassiulas, Distributed algorithms for computation of fair rates in multirate multicast trees, In Proceedings of INFOCOM, Tel Aviv, Israel, 2000.

[117] S. Sarkar and L. Tassiulas, Fair allocation of discrete bandwidth layers in multicast networks, In Proceedings of INFOCOM, Tel Aviv, Israel, 2000.

[118] S. Sarkar and L. Tassiulas, Back pressure based multicast scheduling for fair bandwidth allocation, In Proceedings of INFOCOM, Alaska, 2001.

[119] S. Sarkar and L. Tassiulas, Fair allocation of utilities in multirate multicast networks: A framework for unifying diverse fairness objective, IEEE Transactions on Automatic Control, 47 (6):931-944, 2002.

[120] H. Scarf, The Computation of Economic Equilibria, Yale University Press, New Haven and London, 1973.

[121] J. Shapiro, J. Kurose, D. Towsley and S. Zabele, Topology discovery service for router-assisted multicast transport, In Proceedings of IEEE Open Architectures and Network Programming, 14-24, 2002.

[122] J. K. Shapiro, D. Towsley and J. Kurose, Optimization-based congestion control for multicast communications, IEEE Communications Magazine, 40 (9):90-95, 2002.

[123] T. Sjőstrőm, Implementation in perfect equilibrium, Social Choice and Welfare, 10:97-106, 1993.

[124] V. Smith, Incentive compatible experimental processes for the provision of public goods, In Research in Experimental Economics, Eds.: V. Smith, Greenwich, CT: JAI Press, 1979.

[125] T. Stoenescu, Decentralized Resource Allocation in Networks, PhD thesis, University of Michigan, 2004.

[126] T. Stoenescu, M. Liu and D. Teneketzis, A pricing mechanism for optimal rate allocation in multicast service provisioning, IEEE Transaction in Automatic Control, 2004, submitted.

[127] T. Stoenescu and D. Teneketzis, Minimal message space Nash implementation of unicast resource allocation problems in networks, in preparation.

[128] T. Stoenescu and D. Teneketzis, Informational efficiency of pricing mechanisms in unicast service provisioning, in preparation.

[129] T. Stoenescu and D. Teneketzis, A pricing methodology for resource allocation and routing in integrated-service networks with quality of service requirements, Mathematical Methods of Operations Research (MMOR), 56 (2), 2002.

[130] F. Taylor, The guidance of production in a socialist state, The American Economic Review, 19 (1):1-8, 1929.

[131] P. Thomas and D. Teneketzis, An approach to service provisioning with quality of service requirements in ATM Networks, Journal of High Speed Networks, 6 (4):263-291, 1997.

[132] P. Thomas, D. Teneketzis, and J. MacKie-Mason, A market-based approach to optimal resource allocation in integrated-services connection-oriented networks, Operations Research, 50 (5):603-616, 2002. 
[133] W. Thompson, Concepts of implementation, Japanese Economic Review, 47:133-143, 1996.

[134] H. Tzeng and K. Siu, On max-min fair congestion for multicast ABR service in ATM, IEEE Journal on Selected Areas in Communication, 15 (3), 1997.

[135] F. von Hayek, The nature and history of the problem, In F. von Hayek, Ed., Collectivist Economic Planning: Critical Studies on the Possibilities of Socialism, 1-40, George Routledge \& Sons, London, 1935.

[136] F. von Hayek, The present state of the debate, In F. von Hayek, Ed., Collectivist Economic Planning: Critical Studies on the Possibilities of Socialism, 201-243, George Routledge \& Sons, London, 1935.

[137] F. von Hayek, Economics and knowledge, Economica, 4:33-54, 1937.

[138] F. von Hayek, The socialist calculation: The competitive solution, Economica, 7 (26):125-149, 1940.

[139] F. von Hayek, The use of knowledge in society, The American Economic Review, 35 (4):519-530, 1945.

[140] L. von Mises, Die wirtschaftsrechnung im sozialistischen gemeinwesen, Archiv für Sozialwissenschaft, 47:86-121, 1920.

[141] L. Walras, Éléments d'Économie Politique Pure, ou Théorie de la Richesse Sociale, Paris: Pichon et Durad-Auzias, Lausanne, 1874.

[142] Q. Wang, J. Peha and M. Sirbu, Optimal pricing for integrated-services networks, In L. W. McKnight and J. P. Bailey, Eds., Internet Economics, 353376, MIT Press, Cambridge, MA, 3rd Edition, 1997.

[143] S. Williams, Necessary and sufficient conditions for existence of a locally stable process, Preprint 88, Institute for Mathematics and Its Applications, University of Minnesota, 1984.

[144] E. Zegura, Routing algorithms in multicast switching topologies, In Proceedings of the Allerton Conference on Communication, Control and Computing, Monticello, IL, 1993. 